\title{
Recent Progress in Emerging Near-Infrared Emitting Materials for Light-Emitting Diode Applications
}

\author{
Yingqi Zheng ${ }^{\mathrm{a}, \mathrm{b}}$ (i) \\ Xiaozhang Zhu*a,b (i)

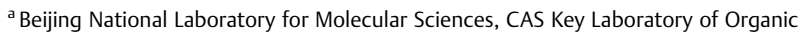 \\ Solids, Institute of Chemistry, Chinese Academy of Sciences, Beijing 100190, China \\ ${ }^{\mathrm{b}}$ School of Chemical Sciences, University of Chinese Academy of Sciences, Beijing \\ 100049, China \\ xzzhu@iccas.ac.cn
}

Received: 16.06 .2020

Accepted after revision: 28.07.2020

DOI: 10.1055/s-0040-1716488; Art ID: om-20-0022rev

License terms: CC)

(c) 2020. The Author(s). This is an open access article published by Thieme under the terms of the Creative Commons Attribution-NonDerivative-NonCommercial-License, permitting copying and reproduction so long as the original work is given appropriate credit. Contents may not be used for commercial purposes, or adapted, remixed, transformed or built upon. (https://creativecommons.org/licenses/by-nc-nd/4.0/).

Abstract In view of the wide applications of near-infrared (NIR) light in night vision, security, medicine, sensors, telecommunications, and military applications, and the scarcity of high-efficiency NIR-emitting materials, development of alternative NIR-emitting materials is urgently required. In this review, we focus on three kinds of emerging NIRemitting materials used in light-emitting diodes (LEDs), namely organic materials, inorganic quantum dot (QD) materials, and organicinorganic hybrid perovskite materials; the corresponding devices are organic LEDs, QD LEDs, and perovskite LEDs. The advantages and disadvantages of the three kinds of materials are discussed, some representative works are reviewed, and a brief outlook for these materials is provided.

Key words near-infrared emitting materials, materials design, organic light-emitting diodes, perovskite light-emitting diodes, quantum dot emitting diodes

\section{Introduction}

ISO 20473 defines infrared (IR) light as an electromagnetic radiation with a wavelength between 780 and $1 \mathrm{~mm}$, which can be divided into three parts: near-IR (NIR; 780-3000 nm), mid-IR (MIR; 3-50 $\mu \mathrm{m}$ ), and far-IR (FIR; 50-1000 $\mu \mathrm{m}) .{ }^{1}$ Almost half the energy reaching the earth's surface from the sun is in the form of NIR radiation. ${ }^{2}$ Thus, materials used in photovoltaics must absorb NIR radiation

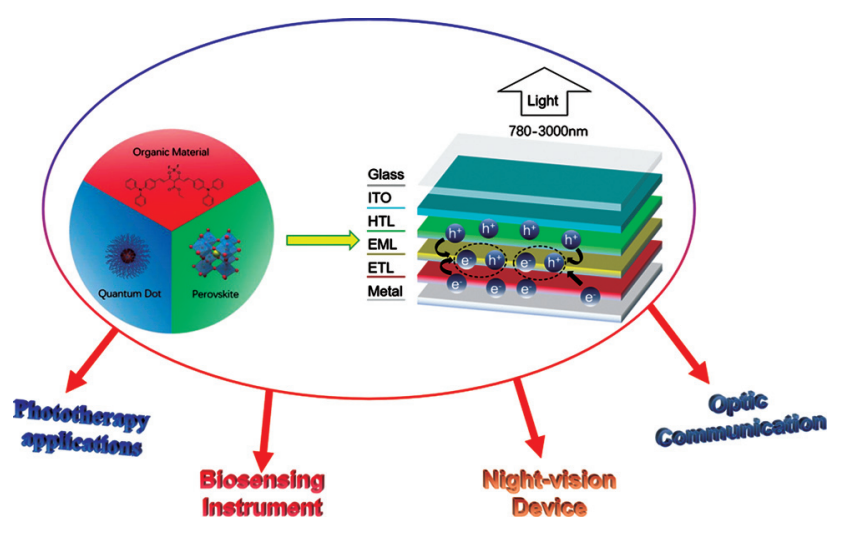

effectively. NIR light has been widely applied in biomedicine, night vision devices, and telecommunications. ${ }^{3-5}$ For example, NIR light is advantageous for biomedical and biosensing applications because of the weak absorption and autofluorescence of biological tissues in the NIR region. Moreover, 700-1000 nm are included in the "semitransparent window" for biological tissues, so it is possible for NIR light-emitting diodes (LEDs) to be integrated into photodynamic therapeutic and bioimaging applications due to the enhanced blood circulation or photoactivation of some drugs by NIR light. ${ }^{6}$ NIR devices can be integrated into through-space optical communication applications, which present the characteristics of anti-interference, security, high-speed, and invisibility as a result of the penetration and independent transmission of IR light. ${ }^{4,5}$ In addition, NIR LEDs are used in biometrics such as identification of finger veins and irises ${ }^{7}$ and in night-vision readable displays. ${ }^{1}$ Thus, development of effective NIR-emitting materials is important for many areas of technology.

There has been much recent progress in the field of LEDs with enhanced performance, and various commercial applications have been developed. Nowadays, many LEDs are based on a structure in which the emitting layer is sandwiched between the cathode and the anode for electron and hole injection. For a multilayer structure, the device may contain electron- or hole-transport layers. Driven by the applied voltage, injected electrons and holes can drift and diffuse in the device, ending up with bounded electronhole pairs, namely excitons, for radiative and nonradiative recombination [Auger recombination or Shockley-ReadHall (SRH) recombination; Figure 1]. There are several key parameters, including turn-on voltage $\left(V_{\mathrm{on}}\right)$, driven voltage, external quantum efficiency (EQE), photoluminescence (PL) quantum yield (PLQY), luminance $\left(\mathrm{cd} \mathrm{m}^{-2}\right)$, and full width at half maximum (FWHM), which serve as figures of merit for 


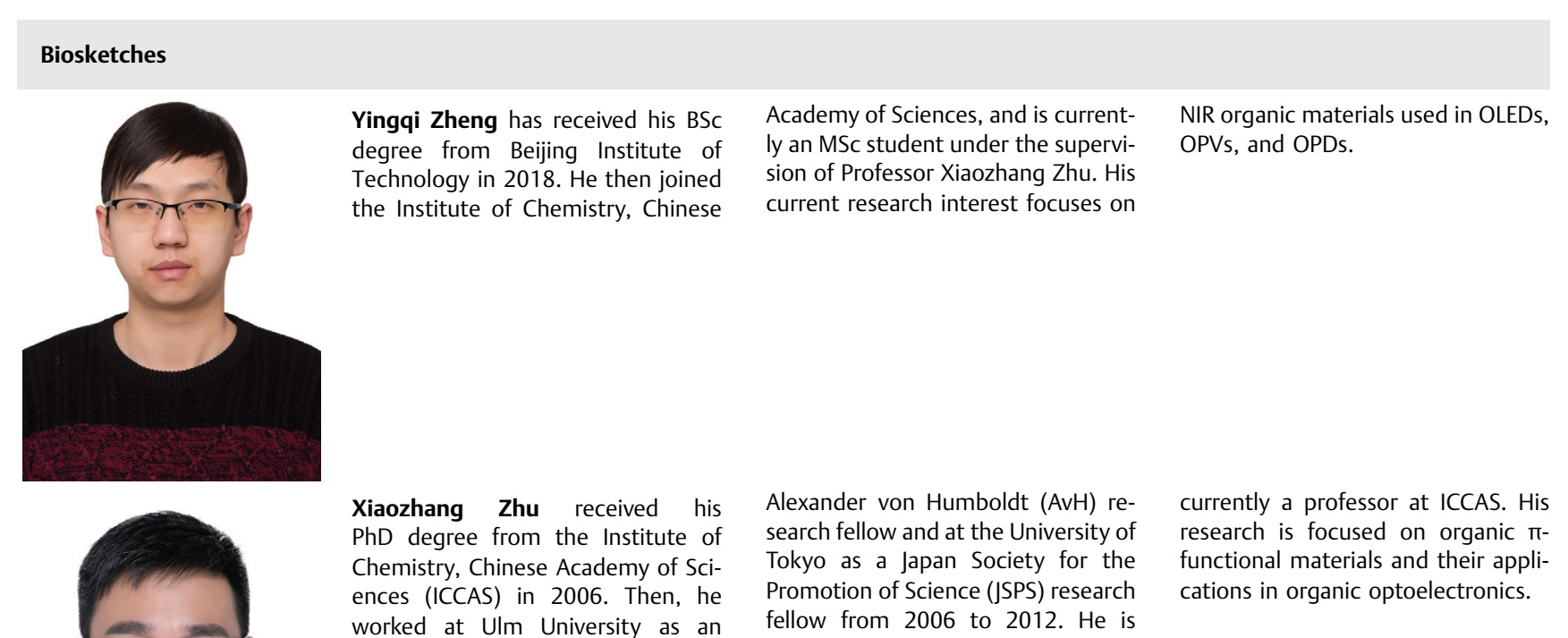

performance evaluation of the LEDs. $V_{\text {on }}$ is the voltage at which the device's luminance achieves $1 \mathrm{~cd} \mathrm{~m} \mathrm{~m}^{-2}$ and the driven voltage is the voltage at which the device is working properly. $V_{\text {on }}$ indicates whether the electrodes contact well with the active layers in the devices and the driven voltage determines the power efficiency (defined as the ratio of the luminance to the driven power, which is the product of the driven voltage and current) and the device lifetime. Furthermore, their luminance and FWHM are important parameters indicating the brightness to the human eye and the color purity of the LEDs, respectively. Different from luminance, radiance ( $\mathrm{W} \mathrm{sr}{ }^{-1} \mathrm{~m}^{-2}$ ) is defined as the flux density of radiant energy per solid angle and per unit of

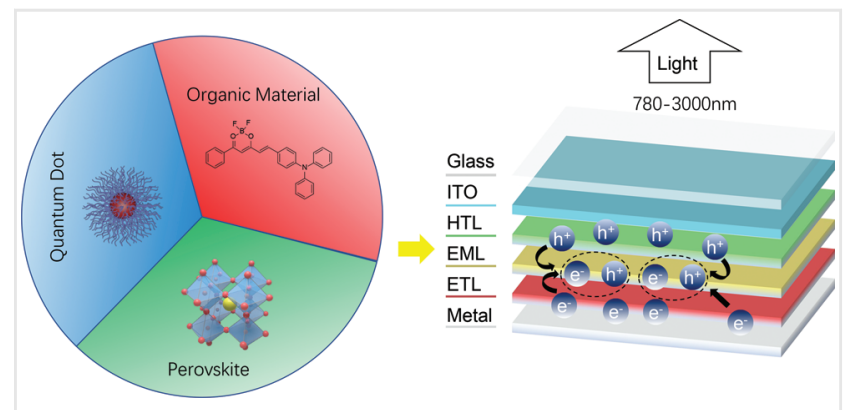

Figure 1 Schematic representation of organic, perovskite, and QD materials and the typical structure of OLEDs, QLEDs, or PeLEDs. projected area of radiating surface and is measured by a machine, not by human eyes. In general, the EQE, defined as the ratio of the number of photons emitted from the device to the number of charge carriers injected into it, is the key parameter for LEDs. Because light can be absorbed, trapped, or reflected by the multiple layers in the device, EQE is the product of light outcoupling efficiency $\left(\eta_{\text {out }}\right)$, namely light extraction efficiency, and the internal quantum efficiency (IQE), defined by the ratio of photons emitted from the emitting layer to the charge injected into the device. Thus, the parameter can be calculated by Eq. (1):

$$
E Q E=\eta_{\text {out }} \cdot I Q E=\eta_{\text {out }} \cdot \gamma \cdot \eta_{r} \cdot \eta_{P L}
$$

where $\gamma$ is the recombination efficiency of injected electrons and holes; in other words, the balance between injected electrons and hole population and under ideal conditions, $\gamma=100 \%$. The term $\eta_{\mathrm{r}}$ can be defined as the radiative exciton ratio in the emitting layer and $\eta_{\mathrm{PL}}$ is the PLQY of the emitting layer. For traditional organic fluorescent materials, $\eta_{\mathrm{r}}(\max )$ is $25 \%$ because of the spin selection rule in the exciton formation process, which states that traditional organic fluorescent materials can only harvest singlet excitons for emission in electroluminescence $(\mathrm{EL}) .^{8}$ However, organic phosphorescent, thermally activated delayed fluorescence (TADF), and emitting materials based on a "hot-exciton mechanism" can make full use of triplet excitons, so their theoretical $\eta_{\mathrm{r}}(\max )$ is $100 \%$. The value of $\eta_{\mathrm{r}}$ is 1 for 
perovskite materials because they are direct-bandgap semiconductors. All these important metrics are used in LED evaluation, and the development of new materials for high-performance LEDs is very important.

Generally speaking, NIR-emitting materials can be roughly classified into inorganic (III-V semiconductors and nanocrystals), organic (small molecules, polymers, organometallic complexes), and hybrid organic-inorganic [quantum dots (QDs), perovskites] materials. In view of the high temperature and vacuum-based processing of the traditional inorganic semiconductors such as the III-V semiconductors, organic materials with easy chemical modification and solution processability, QD materials with high luminescence efficiency and size-dependent emission, and perovskite materials with excellent carriertransport properties have emerged as suitable candidates for NIR-emitting materials. For example, organic materials with nontoxic elements exhibit good biocompatibility, so they can be used in biomedical in vivo applications, ${ }^{6}$ and their solution processability enables large-area fabrication of organic LEDs (OLEDs) when compared with inorganic ones. QD LEDs (QLEDs) are also promising applications for large-area flat-panel displays because of the solution processability, high color purity, and luminescence efficiency of QDs. Considering the high carrier mobility, the low-tomoderate ionization energy, the tunable emission depending on the component ratios, and the two- or threedimensional (2D or 3D) structure of perovskite materials, it is reasonable to assume a luminous future for the application of perovskite LEDs (PeLEDs).

In this review, we focus on three kinds of emerging materials, i.e., organic materials, QDs, and perovskites as emitting layer materials for NIR emission with wavelengths ranging from 670 to $1400 \mathrm{~nm}$. In the following sections, representative published works in the NIR LED region are reviewed, the challenges that have been and need to be addressed are discussed, and an outlook for the developments in these three kinds of materials is presented.

\section{Organic NIR Fluorescent Materials}

Organic materials are widely applied emitting materials because of their tailorable molecular structures, low cost, flexibility, light weight, and tunable emission. To obtain organic materials that emit NIR light, the basic principle is to lower the optical bandgap that is determined by the HOMO and LUMO levels according to molecular orbital theory. The bond-length alternation (BLA) is the average difference in bond length between adjacent single and double $\mathrm{C}-\mathrm{C}$ bonds in a conjugated molecule, which indicates the degree of electron delocalization. The introduction of different electron-rich (electron donor, D) and electron-deficient (electron acceptor, A) units wisely to build a push-pull system is an established approach that is widely used for tuning the molecular energy levels and bandgaps in organic materials due to the hybridization of the energetic levels of the donor and acceptor units and the changed BLA. Generally, in a conjugated system the bandgap narrows when the BLA is reduced by judicious modifications of molecular structures. The introduction and stabilization of quinoidal resonance in aromatic conjugated molecules is also an effective way to reduce the bandgap, which can decrease BLA, and increase the HOMO and lower the LUMO levels. ${ }^{1,9-11}$

Organic conjugated molecules with narrow bandgaps usually have the disadvantage of a relatively strong vibrational coupling between the high vibrational energy levels in the ground state and the low vibrational levels in the first excited state, so the nonradiative transition rate increases exponentially with the decreasing bandgap, which is also called the "energy-gap law." Moreover, due to their extended $\pi$ conjugated lengths, most NIR-conjugated molecules favor a planar structure, which tends to be affected by the aggregation-induced quenching effect. For example, porphyrin is a planar molecule with a four-pyrrole ring and exhibits strong absorption in the red-to-NIR region due to its extended conjugation, but it also presents strong aggregation-induced quenching, which is considered disadvantageous. Intriguingly, in 2001 Tang et al. proposed that molecules with a propellershaped structure illuminate brightly in the condensed state, although in solution the emission is very weak; this effect was named "aggregation-induced emission (AIE)."12

In EL, the ratio of singlet excitons to triplet excitons is $1: 3$ in traditional organic emitting materials due to the spin selection rule. $^{13}$ Thus, the IQE is limited to $25 \%$ in fluorescent OLEDs based on traditional materials because fluorescence originates from the radiative recombination of singlet excitons. To circumvent the drawback, some molecules with new luminous mechanisms were developed, such as TADF, triplet fusion (TF), and doublet emission, which may break the spin selection rule. For OLEDs based on phosphorescent emitters, the radiative exciton ratio can reach up to $100 \%$ in EL. Thus, phosphorescent organometallic materials are alternative choices to avoid the disadvantage and will be discussed in section "NIR Organometallic Phosphorescent Materials." The traditional organic NIR fluorescent materials that obey the spin selection rule and the unconventional organic NIR fluorescent materials that violate the spin selection rule are discussed in the following sections (Table 1).

\section{Traditional Organic NIR Fluorescent Materials Obeying Selection Rule}

In general, traditional organic NIR fluorescent dyes are characterized by being metal-free, environmentally friendly, and cost-effective, and also by their flexibility and 
Table 1 Summary of typical organic NIR-emitting materials and their corresponding EQE values and EL maximums

\begin{tabular}{|c|c|c|c|c|c|c|c|c|c|c|c|}
\hline \multirow[t]{2}{*}{ Emitter } & \multirow[t]{2}{*}{ Host } & \multirow[t]{2}{*}{ EL peak $(n m)$} & \multirow{2}{*}{$\begin{array}{l}\text { Doping } \\
\text { ratio } \\
\text { (wt\%) }\end{array}$} & \multirow{2}{*}{$\begin{array}{l}\mathrm{EQE} \\
(\%)\end{array}$} & \multirow[t]{2}{*}{ Ref. } & & & & (wt\%) & & \\
\hline & & & & & & $\mathrm{T7}$ & DMAC-PN & 730 & 4 & 2.65 & 99 \\
\hline S1 & $\mathrm{Alq}_{3}$ & 752 & 2 & 1.12 & 68 & T8 & CBP & 712 (60 wt\% doped) & 60 & 0.7 & 94 \\
\hline S2 & $\mathrm{Alq}_{3}$ & 748 & 2 & 1.23 & 68 & T9 & $\mathrm{Ga}_{2}(\mathrm{saph})_{2} \mathrm{q}_{2}$ & 700 & 2 & 2.1 & 82 \\
\hline S3 & $\mathrm{Alq}_{3}$ & 823 & 2 & 0.27 & 68 & T10 & & 700 & & 1.54 & 70 \\
\hline S4 & & 690 & & 3.13 & 69 & TTM-3NCz & CBP & 710 (3 wt\% doped) & 3 & 27 & 84 \\
\hline S5 & PIDT-2TPD & 840 & & 1.15 & 33 & TTM-3NCz & CBP & 710 (3 wt\% doped) & 3 & 27 & 84 \\
\hline \multirow[t]{2}{*}{ S6 } & \multirow[t]{2}{*}{$\mathrm{Alq}_{3}$} & \multirow{2}{*}{$\begin{array}{l}749 \\
802\end{array}$} & \multirow[t]{2}{*}{2} & 1.15 & 52 & Po1 & MEH-PPV & 730 & & 0.56 & 18 \\
\hline & & & & 0.43 & 52 & Po2 & PVK:PBD & 720 & 5 & 0.2 & 19 \\
\hline S7 & & 864 & & 0.20 & 52 & Po3 & PVK:PBD & 820 & 2 & 0.10 & 19 \\
\hline S8 & & 706 & & 0.89 & 52 & P6BP & F8BT & 883 & & 0.1 & 20 \\
\hline
\end{tabular}

S9

MEH-PPV/PBD 725

675

F8BT $\quad 720$

S13

S14

S15

S19

S20

P1

P2

P3

P5

P7

P12

P14

P18

P19

P23

P26

$\mathrm{T} 1$

T2

T3

T4

T5

$\begin{array}{lll}\text { T5 } & \text { Zn(BTZ) } & 710(10 \mathrm{wt} \%) \\ & \text { Zn(BTZ) } & 728(20 \mathrm{wt} \%) \\ & \text { ADO-TPA } & 735 \\ & \text { TPBi } & 777 \\ & \text { TPBi } & 693 \\ \text { T6 } & \text { CBP } & 716 \\ & \text { CBP } & 721 \\ & \text { CBP } & 730\end{array}$

Table 1 (Continued)

biocompatibility. Thus, they offer great potential in applications such as bioimaging, night vision, telecommunications, and sensors. ${ }^{2-7,14}$ Based on traditional organic NIR fluorescent materials, the maximum IQE of these devices is limited to $25 \%$ according to the $1: 3$ ratio of singlet to triplet excitons $^{13}$; despite this, the fluorescence lifetimes are of the order of a few nanoseconds, which is ideal for hightransmission-rate applications such as "light fidelity" (Li-Fi), which is a kind of optical communication technique., ${ }^{4,5}$ In this section, we discuss different classes of widely studied NIR fluorescent emitters including porphyrin-based materials, D-A-type small-molecule fluorescent materials, and D-A-type polymeric materials.

\section{Porphyrin-Based NIR Fluorescent Materials}

The first fluorescent OLEDs utilizing conjugated polymers as an active layer were reported by Burroughes et al. in $1990,{ }^{15}$ but only in 1995 were NIR fluorescent OLEDs first reported by Baigent et al. ${ }^{16}$ These latter used a cyanosubstituted thienylene phenylenevinylene copolymer (P1 in Figure 5) as an emitter to fabricate LEDs that show a broad EL spectrum peaked at $740 \mathrm{~nm}$ with a relatively high $\mathrm{EQE}$ of $0.2 \%$ at that time. ${ }^{16}$ After this report, it was found that porphyrin derivatives can be integrated as NIR emitters in fluorescent and phosphorescent OLEDs due to their intense absorption and emission in the red-to-NIR region. ${ }^{17-26}$ Porphyrins, consisting of four pyrrole rings interconnected by ethylene groups and (usually) a central metal ion kept in the center by the correlation bonds with the four $\mathrm{N}$ atoms, are highly stable to light, heat, and $\mathrm{O}_{2}$, and possess excellent fluorescence in solution due to their robust and planar structure. ${ }^{11}$ Moreover, the photophysical properties of porphyrin can be modified by changing substituents or central metal ions.

Grafting the emitting dyes to polymers could be a feasible approach to solving phase separation and 


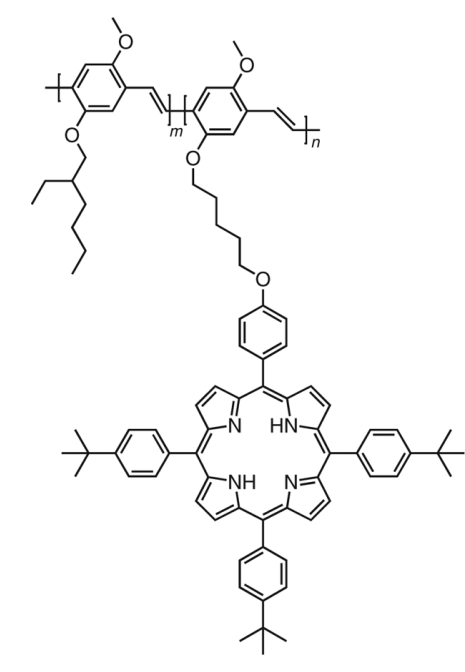

Po1

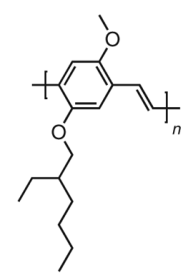

MEH-PPV

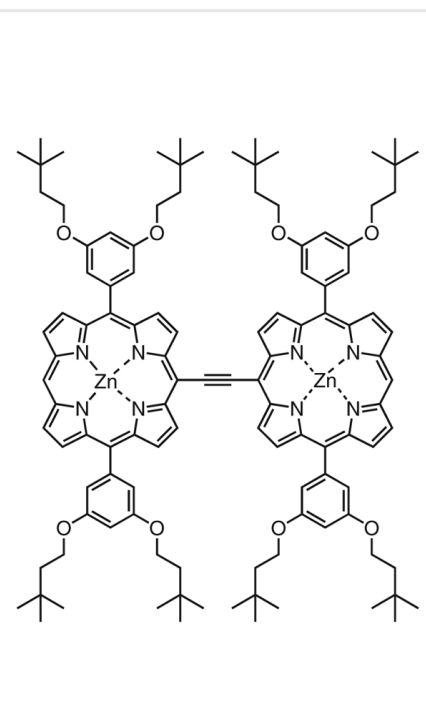

Po2

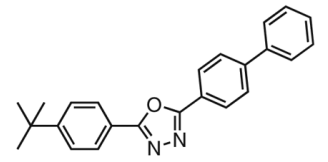

PBD

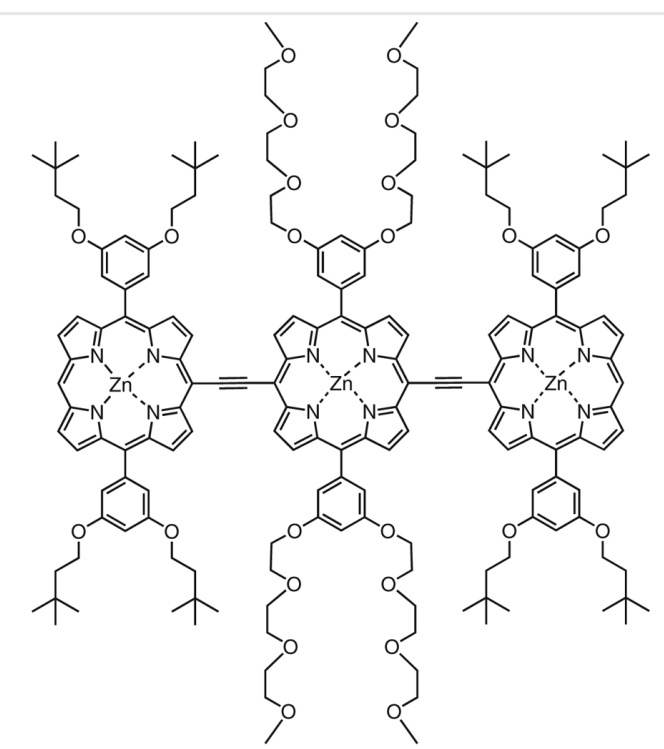

Po3

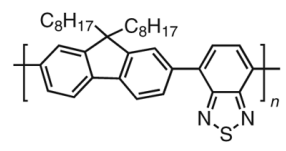

F8BT

Figure 2 Chemical structures of porphyrin-based NIR fluorescent materials and their hosts.

aggregation-induced quenching issues in OLEDs, which may cause poor fluorescence efficiencies in solid-state blending thin films. ${ }^{17,27}$ Polymers with the porphyrin units (Po1 in Figure 2) attached to poly(2-methoxy-5-ethylhexyloxyphenylene vinylene) (MEH-PPV; Figure 2) at different weight loadings (5\% and $8 \%$ ) were synthesized and fabricated as active layers in LEDs by Iqbal et al. in 1999. ${ }^{27}$ The fluorescence of two MEH-PPV porphyrin copolymers was proven to be dominated by the porphyrin units in the PL and EL spectra due to the fast energy transfer from the polymer to the porphyrins. However, the aggregation-induced quenching effect of the porphyrin units arising from the strong intermolecular interaction between chains, which decreases the quantum efficiency of the copolymer, cannot be ruled out completely. Similarly, by tuning the concentration of tetraphenylporphyrin units grafted to MEH-PPV, in 2000, Morgado et al. fabricated some OLEDs based on the same molecule exhibiting emission peaks at 660 and $730 \mathrm{~nm}$ with an EL efficiency of $0.56 \%$. Unfortunately, the tetraphenylporphyrin units grafted to MEH-PPV also suffered severely from the aggregation-induced quenching effect and the limited spectral overlap between the emission band of MEH-PPV and the absorption band of porphyrin units, which reduced the fluorescence efficiency dramatically. ${ }^{18}$

Another polymer-based LED using an ethyne-bridged porphyrin oligomer to construct promising emitters was fabricated by Ostrowski et al. in 2003. ${ }^{19}$ By varying the conjugated length or doping concentration of oligo[(porphinato)zinc(II)] (Po2 and Po3 in Figure 2) in two polymer host materials in the active layer, namely poly $(N$-vinylcarbazole (PVK): 2-(4-biphenyl)-5-(4-tert-butylphenyl)-1,3, 4-oxadiazole (PBD) (60:40 wt\%) and MEH-PPV (Figure 2), they successfully modulated the EL wavelength over a large spectral domain spanning from the low-energy visible region to the high-energy NIR region. The two typical OLEDs exhibit emission peaks at 720 and $820 \mathrm{~nm}$ with an EQE of $0.20 \%$ and $0.10 \%$, respectively. With a similar strategy, Fenwick et al. designed OLEDs incorporating meso-butadiyne-linked linear and cyclic $\mathrm{Zn}$ porphyrin hexamers named P6 and c-P6T (Figure 3) that had redshifted emission ( $\lambda_{\mathrm{PL}}=873$ and $920 \mathrm{~nm}$, respectively) compared to single porphyrin rings by extending their $\pi$-conjugation. ${ }^{20}$ In this work, the authors used a new 
a

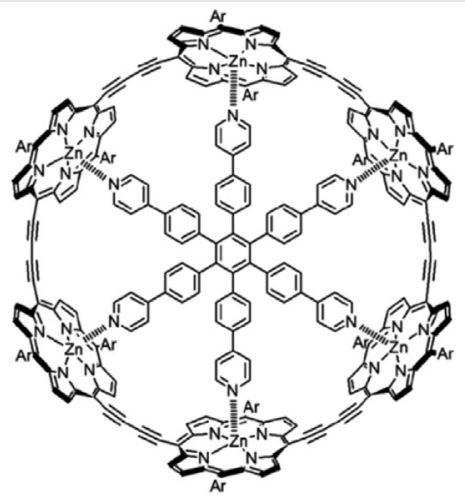

Nanoring, C-P6T

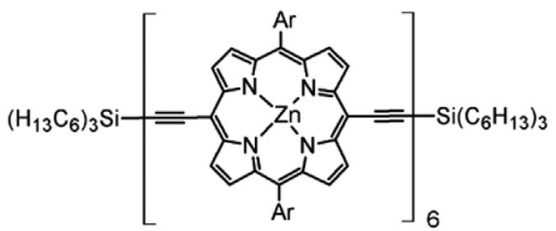

Linear hexamer, P6

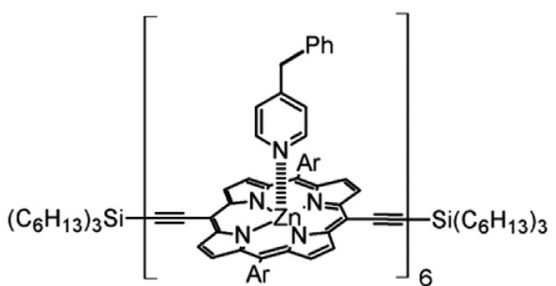

Linear hexamer with 4-benzyl pyridine, P6BP b

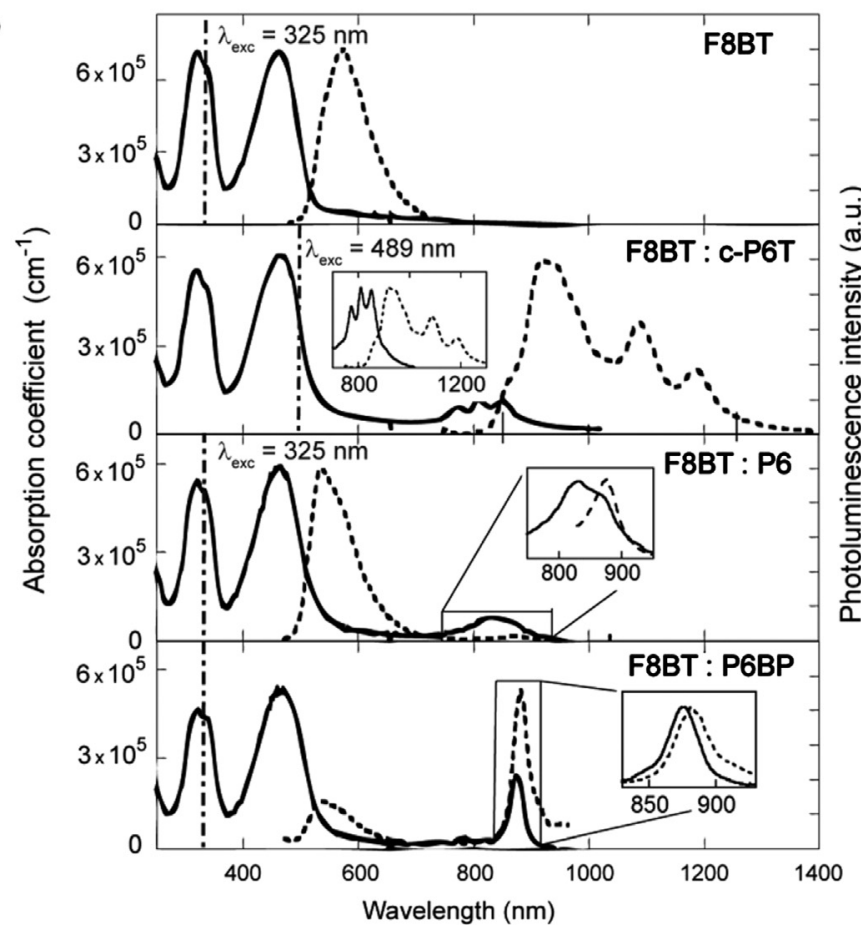

C

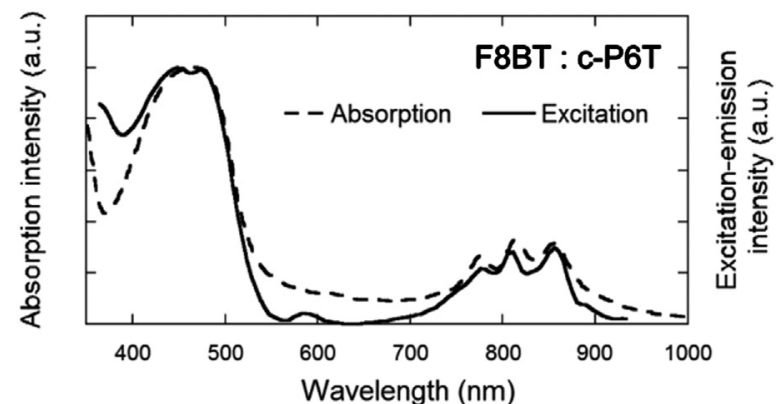

Figure 3 (a) Chemical structures of c-P6T, P6, and P6BP. (b) Absorption (full lines) and PL (dashed lines) spectra of pure F8BT and blend films. Insets show the absorption and emission of the hexamer component of the spectrum. (c) Excitation-emission spectrum of F8BT:c-P6T blends. Reprinted with permission from Ref. 20. Copyright 2011 American Chemical Society.

approach to redshift the EL emission by introducing a cyclic porphyrin hexamer as an emitter in the 9,9'dioctylfluorene-alt-benzothiadiazole (F8BT in Figure 2) host and demonstrated that the curved $\pi$-surface could suppress aggregation. The nanoring $\mathbf{c - P 6 T}$ in the F8BT matrix shed a significantly bathochromic shift with the EL peaking at $960 \mathrm{~nm}$ and the linear P6 in F8BT matrix exhibited an EL peak at $883 \mathrm{~nm}$. Although both devices showed relatively low EQEs of $0.024 \%$ for c-P6T and $0.009 \%$ for P6, the addition of 4-benzyl pyridine to the linear porphyrin hexamer (P6BP in Figure 3) provides additional steric hindrance against aggregation and a weaker intermolecular interaction to provide an order of magnitude increase in EQE up to $0.10 \%$ at $883 \mathrm{~nm}$ in $\mathrm{EL}^{20}$

\section{D-A Type Polymeric NIR Fluorescent Materials}

Among the different organic fluorescent materials investigated so far, the D-A copolymers displayed excellent transport properties and tunable energy levels, and are thus promising candidates for NIR-emitting materials. ${ }^{28-39}$ By appropriately covalent coupling electron-rich $\mathrm{D}$ units to electron-deficient A units to synthesize D-A type molecules, we can narrow the bandgaps of materials to extend the emission in the NIR region, because the emission can be ascribed to the intramolecular charge-transfer state at lower energy compared to localized excited states. ${ }^{1,9-11,29-32}$ The commonly used electron-rich units are thiophene (T), 3,4ethylenedioxythiophene (EDOT), triphenylamine (TPA), fluorene, indacenodithiophene (IDT), indacenodithieno- 


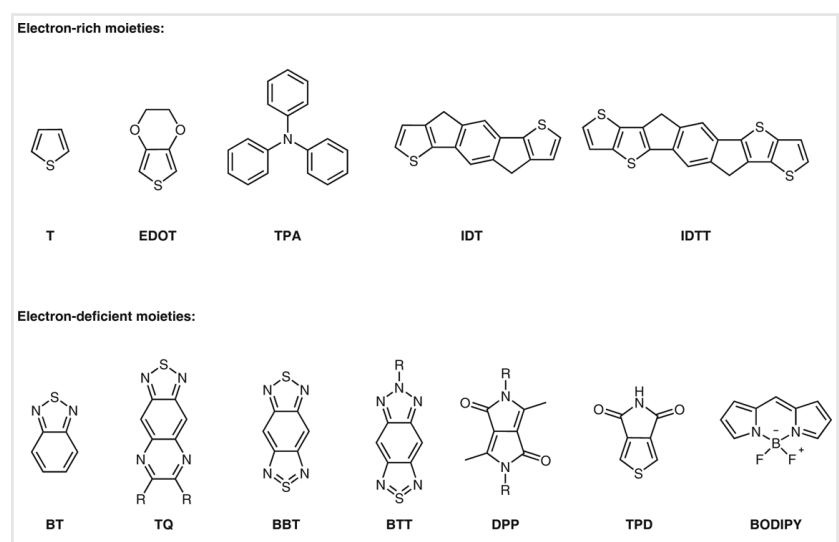

Figure 4 Chemical structures of typical electron-rich and electrondeficient moieties in NIR polymers and small molecules.

thiophene (IDTT), etc., and the commonly used electrondeficient units are benzo[c,1,2,5]thiadiazole (BT), [1,2,5] thiadiazolo[3,4-g]quinoxaline (TQ), benzo[1,2-c:4,5-c]bis $[1,2,5]$ thiadiazole (BBT), [1,2,3]triazolo [4',5':4,5]benzo [1,2-c,1,2,5]thiadiazole (BTT), diketopyrrolo[3,4-c]pyrrole (DPP), thieno[3,4-c]pyrrole-4,6-dione (TPD), boron-dipyrromethene (BODIPY), etc. (Figure 4). ${ }^{34-38,40}$ They all show excellent photophysical and charge carrier-transport properties in organic semiconductor devices such as OLED, organic photovoltaics (OPVs), and organic field-effect transistors. In general, the electron-rich units can raise the materials' HOMO level and the electron-deficient units can lower the LUMO level according to the molecular orbital theory. $^{41}$

Substitution of S by heavier atoms such as Se or Te in the molecular backbone is an alternative way for redshifting the emission wavelength into the NIR region. ${ }^{40,42-47}$ However, the introduction of heavy atoms is usually detrimental to fluorescence emission, as it may cause fluorescent quenching due to enhancement of the spin-orbital coupling effect according to the heavy-atom effect. In 2015, Yang at el. reported on a series of conjugated D-A polymers incorporating poly(9,9-dioctylfluorene) (PFO) as the electron-rich unit and either 4,7-diselenophen-2-yl-2,1,3-benzothiadiazole (SeBT) or 4,7-diselenophen-2-yl-2,1,3-benzoselenadiazole (SeBSe) as the electron-deficient units (P2 and P3 in Figure 5). For the blend of thin films with SeBT or SeBSe loading as low as $1 \%$, the PL emission consisted exclusively of single peaks derived from SeBT or SeBSe units in the copolymers, whereas the emission derived from the fluorene segment was completely quenched. In solution, the emission from the fluorene segment did not disappear instantly with a low content of SeBT or SeBSe units in copolymers and could only be quenched at a certain concentration or content of acceptor units. To shed light on the phenomenon, the authors modulated the copolymer solution concentration and content of the narrow-bandgap units to determine the relative role of the intra- and interchain energy transfer. With the increase in the ratios of narrow-bandgap units, the threshold concentration (the concentration for entirely energy transfer) of copolymers decreased by orders of magnitude. As a result, the efficient energy transfer from the fluorene segment to a SeBT or SeBSe unit proved to be dominated by an intramolecular trapping mechanism. However, the threshold concentrations of PFO-SeBSe in solution for each copolymer were about 5-10 times larger than that of the corresponding PFO-SeBT, which proved that more large-size Se atoms in PFO-SeBSe made the interchain interaction weaker than that in PFO-SeBT. Intriguingly, the copolymer optical bandgap drops dramatically from 2.92 to 1.88 or $1.78 \mathrm{eV}$, respectively, when adding a small amount of SeBT or SeBSe units (5\%) to the main chain. The PL emission peaks of PFOSeBSe were redshifted significantly to the NIR range from 734 for PFO-SeBSe1 (1\%) to $790 \mathrm{~nm}$ for PFO-SeBSe15 (15\%) when compared to the PFO-SeBT counterparts, the emission peaks of which ranged from $671 \mathrm{~nm}$ for PFOSeBT1 (1\%) to 713 for PFO-SeBT50 (50\%). However, the PLQYs decreased from $22 \%$ for PFO-SeBSe1 to only $1 \%$ for PFO-SeBSe. Polymer LEDs (PLEDs) using those emitters delivered a low quantum efficiency of $0.3 \%$ when the EL emission peaked at $723 \mathrm{~nm}$, but the efficiency further decreased to $0.02 \%$ with increasing SeBSe loadings. ${ }^{43}$

Heteroannulation derivatives based on BBT and TQ have been developed as narrow-bandgap guests, the energy levels of which can be well matched with many widebandgap polymers or small molecules. ${ }^{33,48-54}$ In 2015, Tregnago et al. fabricated a series of PLEDs incorporating a bisthienyl(benzotriazolothiadiazole) unit or a bisthienyl (benzotriazoloselenadiazole) unit as the emitter (P4-P7 in Figure 5) with phthalimide-thiophene (P8 in Figure 5) as the host polymer. The EQE of one device with $1 \%$ loading of the bisthienyl(benzotriazoloselenadiazole) unit reached up to $0.018 \%$ with the EL emission peaking at $990 \mathrm{~nm}^{44}$ Similarly, Steckler et al. reported a single-layer NIR PLED with an EQE of $0.27 \%$ at $885 \mathrm{~nm}$ based on phthalimidethiophene copolymerized with bisthienyl(thiadiazoloquinoxaline) or bisthienyl (benzotriazolothiadiazole) as the NIR-emitting segments (P9-P13 in Figure 5). ${ }^{55}$ Therefore, it can be concluded that the heavier atoms extend the emission wavelength to the NIR region and quench the fluorescence concomitantly. ${ }^{43,55}$ In 2007, Sun et al. synthesized a series of conjugated copolymers composed of PFO and 6,7-dimethyl-4,9-di(4-hexylthien-2-yl)-[1,2,5]thiadiazolo[3,4-g]quinoxalines (P14-P17 in Figure 5) by Pdcatalyzed Suzuki coupling reactions. PLEDs based on these copolymers showed NIR emission between 800 and $900 \mathrm{~nm}$ and the highest EQE reached up to $0.30 \%$ at $824 \mathrm{~nm}$ from PFO-DDTQ1-based devices. ${ }^{49}$ In 2015, Marder et al. investigated heteroannulated acceptors based on benzothiazole 
and authored a comprehensive review which offered interesting insights into the electronic properties and structures of these materials. $^{36}$

Recently, DPP derivatives have drawn much attention because of their wide applications in laser printers, information storage systems, erasable optical memory devices, and emitting layers. ${ }^{40,52-54,56-60}$ In 2002, Beyerlein et al. reported on a new hairy rod-type conjugated polymer consisting of N-alkylated diketopyrrolopyrrole (DPP) diphenylene and alkoxy-substituted phenylene units in the backbone (P18 in Figure 5) featuring a characteristic bright-red PL peaking at ca. $640 \mathrm{~nm}$. The devices based on this material exhibited an EL emission peaking at $650 \mathrm{~nm}$ and a maximum EQE of $0.7 \%$ with a $V_{\text {on }}$ of $5 \mathrm{~V}$, although they suffered from a severe efficiency roll-off at high voltages. Their EQE dramatically dropped from $0.7 \%$ to $0.4 \%$ with a change in the applied voltage from 5 to $20 \mathrm{~V} \cdot{ }^{61}$ After a few years, F8BT (Figure 2) was proved to behave as the best host polymer in view of previous NIR OLED reports and the efficiency roll-off could be effectively solved by copolymerizing DPP-based D-A-D units with F8BT. ${ }^{20,39,62-65}$ In 2013, Fenwick et al. reported two efficient deep-red emitters, DPP1 and DPP3 (1\% and 3\% correspond to the initial monomer ratios), consisting of a small number of DPPbased moieties copolymerized with F8BT (P19 in Figure 5), which reached up to a $1 \%$ EL quantum efficiency at $670 \mathrm{~nm}$ and about $67 \%$ solid-state PL quantum efficiency. The efficiency roll-off effect, which arises from excess unbalanced carriers passing through the devices and then recombining at the anode or cathode, could be eliminated by replacing the hole-transport layer poly(3,4-ethylenedioxythiophene) (PEDOT:PSS) with an electron-blocking

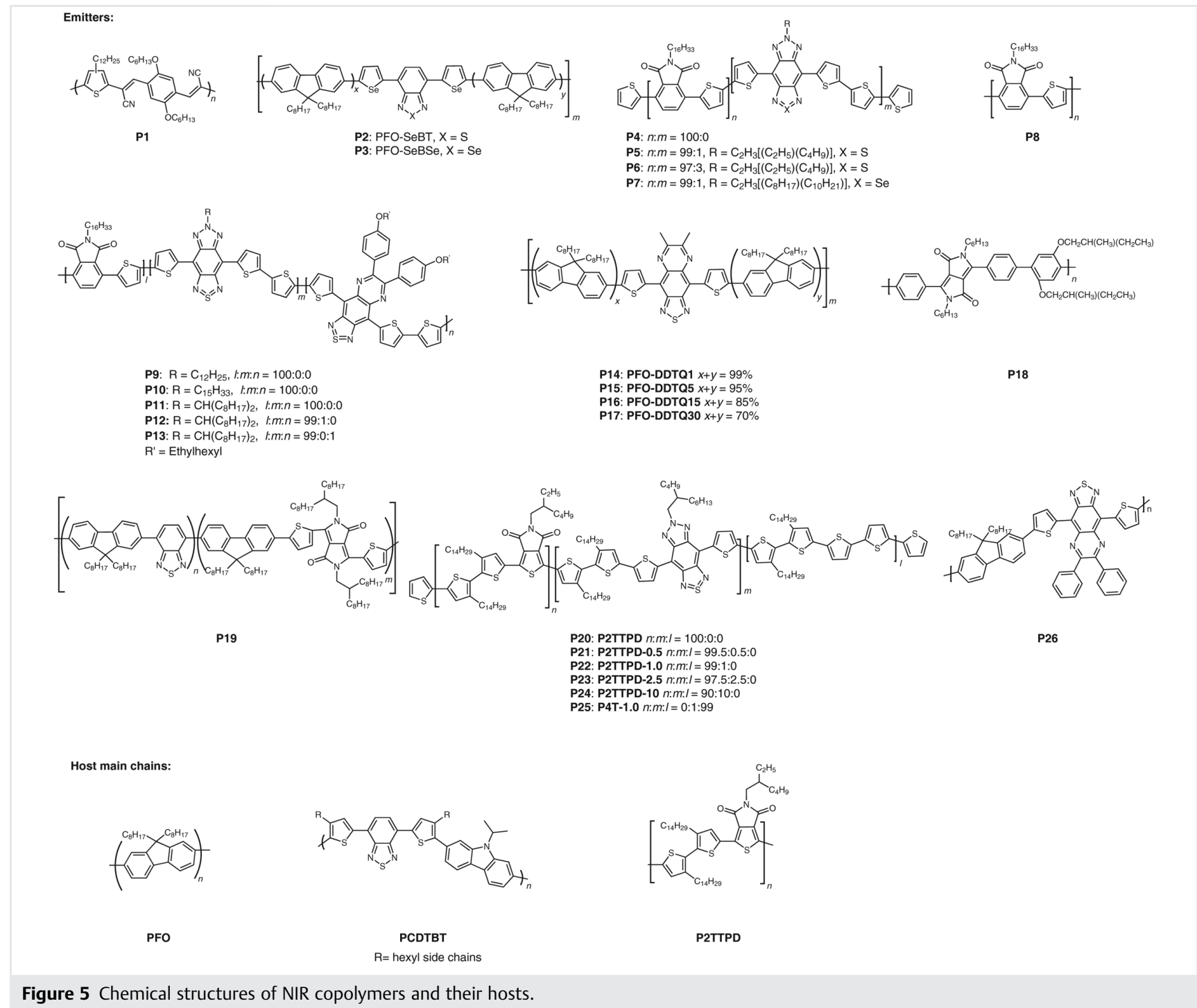


poly(9,9'-dioctylfluorene-alt- $N$-(4-butylphenyl)-diphenylamine) (TFB) interlayer. ${ }^{39}$

Another intriguing NIR-emitting D-A alternating copolymer, poly[ $N$-9'-heptadecanyl-2,7-carbazole-alt-5,5-(4',7'-di2-thienyl-2', $1^{\prime}, 3^{\prime}$-benzothiadiazole) (PCDTBT in Figure 5) as the polymer main chain incorporating thiophene-benzothiadiazole-thiophene (TBT) as electron-deficient units and carbazole $(\mathbf{C z})$ as electron-rich units was reported by Lombeck et al. in 2016. They introduced a side-chaincontrolled strategy to modulate the copolymers' solubility and photophysical properties by the addition of a hexyl side chain to TBT units. With increasing amounts of hex-TBT units from $0 \%$ to $100 \%$ in the copolymer, the backbone twisted, and the absorption spectrum shifted to the blue region. Both the PL and EL quantum efficiencies increased with increasing hex-TBT content (which was of considerable interest). It was shown that the EL quantum efficiency of the fully hexylated PCDTBT reached up to $1.1 \%$ with the EL peaking at $680 \mathrm{~nm}$, and with luminance up to $2500 \mathrm{~cd} \mathrm{~m}^{-2}$, it was enhanced by 2 orders of magnitude compared to standard PCDTBT. ${ }^{66}$ To extend the emission of materials further into the NIR region, Zampetti et al. reported a new series of copolymers based on the triazolobenzothiadiazole (BTT) derivatives 6-(2-butyloctyl)-4,8-di(thiophen-2-yl)-[1,2,3]triazolo[4',5':4,5]benzo

$[1,2-c]-[1,2,5]$ thiadiazole (TBTTT) as emitters included in the D-A copolymer (P20-P25 in Figure 5), with alternating bithiophene and thienopyrroledione (TPD) moieties (P2TTPD in Figure 5) as the host matrix. Intriguingly, the PLEDs fabricated with the pure host polymer had a maximum EQE of $0.49 \%$ at $690 \mathrm{~nm}$ with a $V_{\text {on }}$ as low as 2.4 V. NIR emission peaking at about $900 \mathrm{~nm}$ with EQE up to $0.15 \%$ was obtained by P23-based PLEDs with a $V_{\text {on }}$ of only $5 \mathrm{~V}$. In this work it was demonstrated that such a low $V_{\text {on }}$ could be attributed to the efficient intrachain energy transfer to the TBTTT moieties. ${ }^{35}$

As discussed above, copolymerizing NIR moieties with a wide-bandgap polymer host main chain is a feasible way of avoiding the aggregation-induced quenching effect and reduces phase segregation to some extent. However, copolymerization of emitters and host main chain seems to be a better approach to NIR emission and effective luminescence than blending, but there still are some drawbacks. The disadvantages are as follows: (i) chromophore aggregation and close packing due to either interchain or intrachain interaction still take place in copolymers. It is hard for us to determine and control the distribution of different moieties in each polymer chain for the copolymer and the exact unit amount for each chain; (ii) by copolymerization, the frontier orbital energy level may change for each moiety; thereby, the formed heterojunction may not end up being a type-I heterojunction, which is commonly preferred to energy transfer over charge transfer; (iii) the copolymerization of D and A units is more costly than the blend-based method. ${ }^{67}$

\section{D-A-Type Small-Molecule NIR Fluorescent Materials}

The same $\mathrm{D} / \mathrm{A}$ building blocks mentioned above can be combined to synthesize D-A-D or A-D-A small molecules as emitters in active layers. ${ }^{33,51,52,64,68-71}$ In 2009, Qian et al. reported three kinds of D-A-D chromophores based on TQ as an electron-deficient unit and TPA as electron-rich units (S1-S3 in Figure 6). By changing the electron-rich substituted units on the TQ core, they successfully fabricated OLEDs with tunable EL peaks ranging from 768 to $870 \mathrm{~nm}$. The best OLED based on 2 wt\% $\mathbf{S 2}$ doped in Alq $\mathbf{3}$ (Figure 6) showed an exclusive NIR emission at $748 \mathrm{~nm}$ with an EQE of $1.23 \%$ over a wide range of current density, and a maximum radiance of $2880 \mathrm{~mW} \mathrm{Sr}^{-1} \mathrm{~m}^{-2}$ at $15 \mathrm{~V}^{68}$ Ledwon et al. reported a new organic material with the $D-\pi-A-\pi-D$ structure utilizing $\mathbf{C z}$ as the electron donor and benzothiadiazole as the electron acceptor (S4 in Figure 6). A strong solvatochromic effect in its EL spectrum and the electron paramagnetic resonance demonstrated its intramolecular charge-transfer property and the delocalization of radical cations and anions over the molecular backbone. OLEDs based on $\mathbf{S 4}$ exhibited an EL peak at $690 \mathrm{~nm}$ with a $V_{\text {on }}$ of $4 \mathrm{~V}$, a maximum EQE of $3.13 \%$ that was a relatively high value among other $\mathbf{C z}$-based and benzothiadiazole-based materials. $^{69}$

Blending of an appropriate host and emitter to produce a thin film as the emitting layer is a practical method of highefficiency OLED fabrication. Thus, the selection of matched host and emitter becomes crucial in the OLED fabrication process. In 2018, Minotto et al. reported on a new electroluminescent blend film incorporating $\mathbf{S 5}$ as the emitter and PIDT-2TPD (Figure 6) as the host matrix. OLEDs based on the blend films afford excellent spectral and transport properties such as the EL peaking at $840 \mathrm{~nm}$, a $V_{\text {on }}$ of $1.7 \mathrm{~V}$, and an EQE up to $1.15 \%$. The improved device performance was attributed to the good spectral overlap and charge transfer in the D-A-D structure of S5 and the high PLQYs of both PIDT-2TPD and S5. The host matrix, PIDT-2TPD, contains an IDT as the electron-rich unit and two TPDs as the electron-deficient units in each monomer. The presence of two TPD units was beneficial: a good electron-transport property of PIDT-2TPD, good spectral overlaps between their photoemission and the absorption of $\mathbf{5} 5$ were achieved (better than F8BT), and the PLQY of the thin film (PIDT-2TPD doped with 0.5 wt\% S5) attained $18 \%$. As a consequence of the excellent spectral overlap between the absorption of $\mathbf{S 5}$ and the emission of PIDT-2TPD and their high PLQYs, the maximum EQE of $1.15 \%$ is the highest among the reported undoped devices, peaking at around $840 \mathrm{~nm}$. To the best of our knowledge, the device performance is the best ever reported for the EL wavelength above $800 \mathrm{~nm}$ with a purely organic and solution-processed active layer, not leveraging a triplet-assisted emission. ${ }^{33}$ In 2012, a family of D-A-D type NIR fluorophores containing nonplanar conjugated tetraphenylethene (TPE) moieties was developed with electron- 


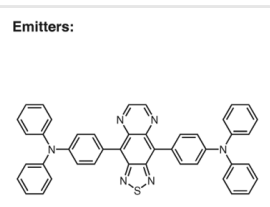

s1

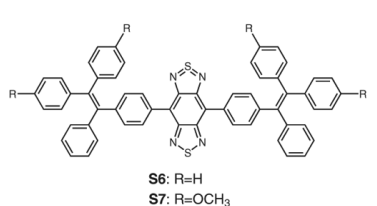

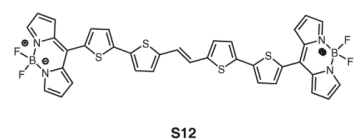

S12

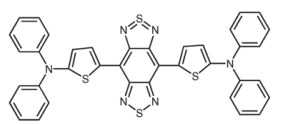

$\mathbf{S 1 6}$

Hosts:

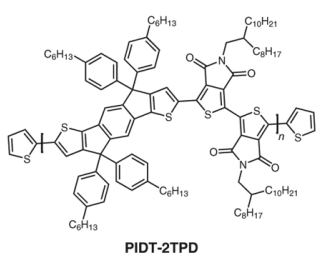

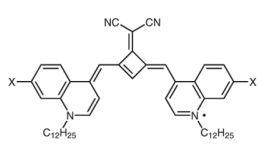

s17 $\mathrm{X}=\mathrm{F}, \mathrm{Cl}, \mathrm{Br}, \mathrm{I}$

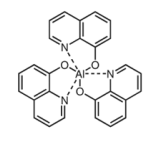

$\mathrm{Alq}_{3}$

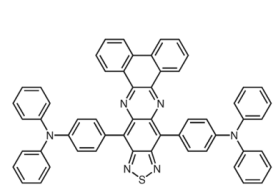

s3
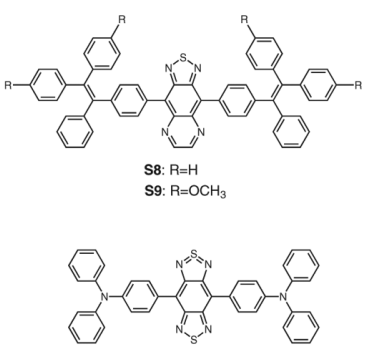

S13

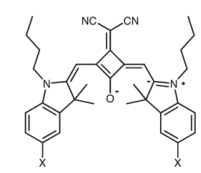

$S 18$
$X=F, C l, B r, I$

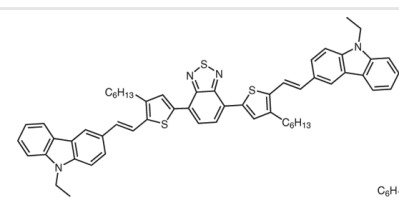

s4
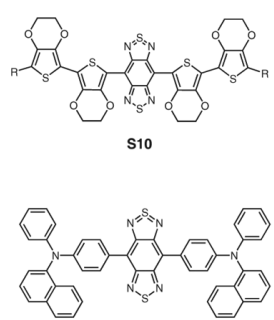

S14
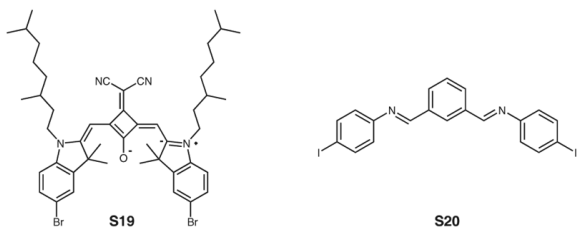

S20

Figure 6 Chemical structures of NIR small molecules and their hosts.

deficient TQ or BBT as A units (S6-S9 in Figure 6). The twisted TPE units that restrict the intramolecular rotation and reduce the $\pi-\pi$ stacking give rise to a good AIE enhancement (AIEE) property. The PL spectra of these fluorophores range from 600 to $1100 \mathrm{~nm}$ with their optical bandgaps ranging from 1.85 to $1.50 \mathrm{eV}$. Undoped OLEDs based on these fluorophores exhibited an EL emission peaking from 706 to $864 \mathrm{~nm}$ with the EQEs spanning from $0.89 \%$ to $0.20 \%$. This work demonstrated that compounds with an AIEE property are suitable for fabricating efficient undoped NIR OLEDs. ${ }^{52}$ Another series of multi-heterocycle D-A-D telechelic-conjugated oligomers, based on electron-rich thiophene, phenylene, and EDOT units as donor units along with electron-deficient benzothiadiazole as acceptor units (S10 in Figure 6), were designed and investigated by Ellinger et al. in 2011. These authors observed that $\mathbf{S 1 0}$ has a strong tendency to aggregate regardless of the host matrix. By blending the oligomer
S10 in a MEH-PPV/PBD matrix, the EQE of the OLEDs achieved $\sim 0.5 \%$ with an EL peak at $725 \mathrm{~nm}^{72}$

Impressive D-A type chromophores may guide scientists to find new luminous mechanisms and design a strategy for organic materials. In 2015, Han et al. reported a new D-A-D type NIR fluorescent compound, 2,3-bis(4'-(diphenylamino)-[1,1'-biphenyl]-4-yl)fumaronitrile (S11 in Figure 6), that also exhibited strong NIR fluorescence with a corresponding thin-film quantum efficiency of 33\% due to its "hybridized local and charge transfer" (HLCT) and AIE property. Undoped and metal-free OLEDs based on the $\mathbf{S 1 2}$ feature a high maximum EQE of $2.58 \%$ and the EL emission peaked at $675 \mathrm{~nm}$ with a $V_{\text {on }}$ as low as $3.6 \mathrm{~V} .^{71}$ In 2017 , a new A-D-A oligomer S12 (Figure 6) incorporating two $\alpha, \beta$ unfunctionalized 4,4-difluoro-4-bora-3a,4a-diaza-s-indacene (BODIPY) as A units and two ethylene-bridged bithiophenes was synthesized and characterized by 
Zampetti et al. $^{64}$ Because of the delocalization of the BODIPY low-lying LUMO over the oligothienyl moieties (demonstrated by density functional theory [DFT]), the PL emission extends to the NIR region with a PL efficiency of $20 \%$ in the solid state. According to the DFT calculations, such an intramolecular energy structure should favor a "hole funneling" effect towards the central bithienyl moieties in the presence of a substantially homogeneous distribution of electrons on a relatively low-lying LUMO, which could be effectively populated from a host polymer such as F8BT in the guest-host systems. In this regard, the authors blended F8BT as the host matrix with S12 as the emitter to form a straddling heterojunction to localize the exciton in $\mathbf{S 1 3}$ for the following radiative decay. PLEDs based on S12/F8BT blends exhibited an EL that peaked at $720 \mathrm{~nm}$ with a maximum EQE of $1.1 \%$. The two examples prove that the A-D-A molecules are promising candidates for NIR light-emitting materials. ${ }^{64}$

Although much progress has been made, there are still numerous challenges in regard to NIR emission above $1000 \mathrm{~nm}$. A representative work was reported by Qian et al., who synthesized a series of simple NIR D-A-D molecules consisting of benzo(1,2-c:4,5-c)bis((1,2,5)thiadiazole) (BBTD) as the acceptor and four different donor moieties (S13-S16 in Figure 6). The OLEDs based on these materials exhibit EL emission peaks ranging from 1050 to $1220 \mathrm{~nm}$, with a maximum EQE up to $0.28 \%$ at a current density of $10 \mathrm{~mA} \mathrm{~cm}^{-2} .51$

Squaraine dyes have drawn much attention because of their sharp and intense absorption in the long-wavelength region, and the good photostability from their rigid structure. $^{28,73}$ In 2012, Würthner and coworkers reported bright NIR fluorophores based on squaraine with an unexpected halogen effect that violates our conventional perception. In their work, the introduction of halogen atoms induced an increase in the molar absorption coefficient $(\varepsilon)$ and a redshift that increased in the sequence of $\mathrm{H}, \mathrm{Cl}, \mathrm{Br}$, I-substituted squaraine derivatives (S17 and S18 in Figure 6). The spectral emission region of three kinds of halogen-substituted squaraine derivatives could be extended to above $900 \mathrm{~nm}$. They attributed the halogen effect to an electron density transfer from the substituents to the chromophore core and a strengthened conjugation along the $\pi$ system. With increasing polarizability of halogen substitutes, the halogen effect became more pronounced. This work proved that squaraine dyes have a promising prospect for NIR luminescence. ${ }^{73}$ In 2013, Stender reported a solution-processible NIR OLED consisting of an organic host-guest system, incorporating SY-PPV (Figure 6) as the host and a bromoindolenine squaraine dye $\mathbf{S 1 9}$ (Figure 6) as the guest, featuring a highly efficient energy transfer from the visible spectral range to the NIR (about $750 \mathrm{~nm}$ ). The OLED with a doping concentration of $0.05 \mathrm{wt} \%$ achieved a maximum EQE of $0.65 \%$ with EL emission peaking at $730 \mathrm{~nm}^{28}$

\section{Unconventional Organic NIR Fluorescent Materials Breaking the Selection Rule}

Much progress has been achieved in recent years toward development of materials for OLEDs due to the tunability of organic materials by molecular tailoring. In typical OLEDs, holes and electrons injected from the anode and cathode respectively combine in the emitting layer to form singlet and triplet excitons at a ratio of 1:3 according to the spin statistics. $^{13,74}$ Therefore, the IQE of OLEDs based on conventional organic materials is limited to $25 \%$. TADF, which was also called "E-type delayed fluorescence (DF)," was first rationalized by Perrin in $1929 .{ }^{75}$ Furthermore, the so-called E-type DF was also reported in eosin and benzil by Parker and Hatchard, respectively. ${ }^{76,77}$ The TADF mechanism was recently reinvigorated by Adachi, who fabricated OLEDs that exploit nonradiative triplet excitons and thus the overall EL efficiency is enhanced. The energy gap of organic materials between singlet and triplet excited states can be lowered. Typically, TADF organic molecules are characterized by a highly efficient spin upconversion from nonradiative triplet states to radiative singlet states with a high radiative decay rate exceeding $10^{6}$ decays per second. Therefore, the molecules can harvest both singlet and triplet excitons for EL and even feature relatively high IQEs exceeding $90 \% .^{78-81}$ To date, this mechanism has been studied extensively, and in this section we will focus on TADF NIR fluorescent materials. In addition, triplet-triplet annihilation (TTA), by which two triplet excitons fuse into one singlet exciton, is an alternative way of fully utilizing triplet excitons for fluorescence emission. ${ }^{80,82}$ Fluorescence emission from radicals' doublet excitons can be another way to make full use of excitons in EL. ${ }^{83,84}$

\section{TADF NIR-Emitting Materials}

To narrow the energy gap of singlet and triplet excited states in organic molecules, many researchers combined strong D and A moieties to obtain TADF molecules for the formation of intramolecular charge transfer (ICT) in excited states. The general TADF molecular design strategy is to spatially separate D and A moieties bridged by aromatic units or to twist the $\mathrm{D}$ and $\mathrm{A}$ moieties in the molecular backbone to localize LUMO in A moieties and HOMO in D moieties to limit the overlap between HOMO and LUMO in ICT excited states. ${ }^{80,81,85-88}$ A poor overlap between LUMO and HOMO leads to a small singlet-triplet energy gap, as well as to a lower oscillator strength, which may decrease the radiative rates according to Fermi's golden rule. ${ }^{78}$ Due to the limitation of the intrinsic energy-gap law and the aggregation-induced quenching effect, the NIR OLEDs based on TADF emitters have to date seldom exhibited EQE values above 5\%. Nevertheless, EQE values close to $30 \%$ were achieved for the red TADF OLEDs ${ }^{89,90}$ and the first deep-red 

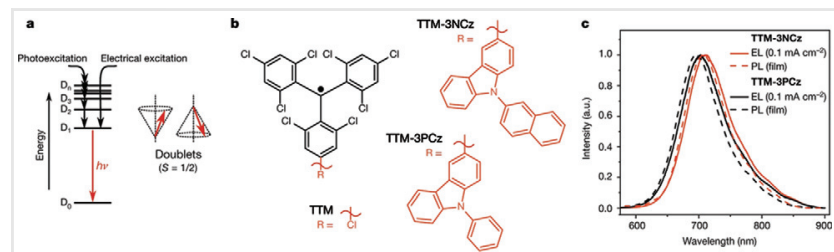

Figure 7 (a) Doublet emission after photo- or electrical excitation. The right-hand illustration shows the electron spin vector directions for doublets. (b) Chemical structures of TTM, TTM-3NCz, and TTM-3PCz. (c) EL (solid lines) and PL (dotted lines) spectra for TTM-3NCZ (red) and TTM-3PCz (black) LEDs. Reprinted with permission from Ref. 84. Copyright 2018 Springer Nature.

TADF OLED with an EQE value of nearly $10 \%$ and an EL that peaked at $668 \mathrm{~nm}$ was first reported by Wang et al. with a novel NIR TADF V-shaped $D-\pi-A-\pi-D$ molecule T1 (Figure 8) with a small singlet-triplet splitting $\left(\Delta E_{\mathrm{ST}}\right)$ of $0.13 \mathrm{eV}$ originating from its ICT feature and rigid structure. The optimized OLEDs, based on blends of 1,3,5-tris(Nphenyl-benzimidazol-2-yl)benzene (TPBi in Figure 8) as the host matrix and T1 as the dopant, exhibited a maximum EQE value of $9.8 \%$ with peak EL emission at $668 \mathrm{~nm}$. Intriguingly, they demonstrated that $\mathbf{T 1}$ is also an AIE-active material with increased fluorescence quantum efficiencies in the solid state as compared to solution due to its twisted structure. The undoped devices achieved a maximum EQE of $2.1 \%$ because of its high PLQY in the solid state. These deepred materials laid a good foundation for the further study of NIR materials. ${ }^{91}$ Two years later, Wang et al. reported NIR OLEDs based on the TADF emitter T2 (Figure 8) doped in TPBi and exhibiting a high EQE of $14.5 \%$ at $644 \mathrm{~nm}$ and $3.9 \%$ at 728. Through X-ray diffraction (XRD) analysis, they demonstrated an edge-to-edge aggregation in the crystal of T2; it shows a strong deep-red or NIR emission in a neat thin film $\left(\lambda_{\max }=733 \mathrm{~nm}\right.$, PLQY $\left.=21 \%\right)$. This work demonstrated that a rational design of a supramolecular structure of a molecule is a feasible way to synthesize high-efficiency NIR organic materials. ${ }^{87}$

In 2017, two dendrimer-type NIR TADF emitters, 2,6-bis (4-(bis(4-((6-(9H-carbazol-9-yl)hexyl)oxy)phenyl)amino) phenyl)anthracene-9,10-dione (T3 in Figure 8) and 2,6-bis (4-(bis(4-((6-(9'H-[9,3':6',9'"-terbenzo[b]indol]-9'-yl)

hexyl)oxy)phenyl)amino)phenyl)anthracene-9,10-dione (T4 in Figure 8), were reported by Sun et al. and the undoped OLEDs based on $\mathbf{T} 4$ exhibited a maximum EQE of $0.254 \%$ with EL peaked at $715 \mathrm{~nm}$. The dendrimers exhibit a self-host feature and the peripheral $\mathrm{Cz} /$ tricarbazole dendrons encapsulate the core to suppress aggregation. ${ }^{86}$ Furthermore, in the same year, Yuan et al. reported a wedge-shaped $D-\pi-A-\pi-D$ TADF emitter 3,4-bis(4-(diphenylamino)phenyl)acenaphtho [1,2-b]pyrazine-8,9-dicarbonitrile (T5 in Figure 8) with a small singlet-triplet splitting $\left(\Delta E_{\mathrm{ST}}\right)$ of $0.14 \mathrm{eV}$, synthesized through combining an acenaphtho[1,2-b]pyrazine-8,9-dicar- bonitrile acceptor core with twoTPA donor units. The undoped NIR devices featured a maximum EQE of $2.19 \%$ with the EL emission peaking at $777 \mathrm{~nm}$, whereas the doped devices based on blends of a TPBi host matrix doped with $10 \mathrm{wt} \%$ of T5 were characterized by an extremely high EQE of $10.19 \%$ and an EL peak at $693 \mathrm{~nm}$. The high quantum efficiencies were attributed to a moderate orbital overlap between the HOMO and the LUMO in $\mathbf{T 5} .^{92}$ The same group later reported a series of T5-based OLEDs by varying the host materials such as ADOTPA (Figure 8) for adjusting the neighboring surroundings. It turned out that an OM compound bis[2-(2-benzothiazolylN3)phenolato-O]zinc ( $\mathbf{Z n}(\mathbf{B T Z})_{\mathbf{2}}$ in Figure 8 ) as the host material matched best with $\mathbf{T 5}$ and the doped devices achieved rather high EQE values of $12.3 \%$ and $5.1 \%$ by varying the doping concentration from $2 \mathrm{wt} \%$ to $20 \mathrm{wt} \%$, and EL peaks ranging from 668 to $728 \mathrm{~nm}$. The relatively high fluorescence efficiencies of the doped films can be ascribed to the sufficient spectral overlap between the emission spectrum of $\mathbf{Z n}(\mathbf{B T Z})_{\mathbf{2}}$ and the absorption spectrum of $\mathbf{T 5}$. The redshifted emission peaks can be ascribed to the solid-state solvation effect of $\mathbf{Z n}(\mathbf{B T Z})_{\mathbf{2}}$ because of the largest dipole moment among all the used hosts. ${ }^{93}$

In contrast to the reported twisted TADF molecules, curcuminoid derivatives with no twisted structure exhibited exceptional photophysical properties in OLED applications. In 2018, Kim et al. reported on two D-A-D-type boron difluoride curcuminoid derivatives consisting of one or two triphenylamine donor groups and one acetylacetonate boron difluoride acceptor unit as effective TADF emitters (T6 and T8 in Figure 8). ${ }^{94,95}$ For T6, unlike the general strategy to obtain TADF molecules with the spatially separated HOMO and LUMO, the large spatial overlap between the hole and electron wave functions of the boron difluoride curcuminoid derivative $\mathbf{T 6}$ gives rise to a high molar extinction coefficient and oscillator strength, which positively correlate with the high radiative decay rate, rendering it a good emitting material for OLEDs. Interestingly, an efficient TADF activity was observed in its thin film. Moreover, its absorption and emission bands can be tuned in a broad range due to the large ground- and excited-state dipole moments. Quantum-chemical calculations demonstrated that the vibration and spin-orbit coupling assisted TADF mechanism involved in $\mathbf{T} \mathbf{6}$ can be attributed to an enhanced nonadiabatic coupling effect between the lowlying excited states. In conclusion, the NIR OLEDs based on blends of the 4,4'-di(9H-carbazol-9-yl)-1,1'-biphenyl (CBP in Figure 8) host and the curcuminoid derivative T6 exhibited a maximum EQE of nearly $10 \%$ at $721 \mathrm{~nm}$ and a maximum radiance value of $3 \times 10^{6} \mathrm{~mW} \mathrm{sr}^{-1} \mathrm{~m}^{-2}$. By modulating the polarity of the active medium, the EL peaks can be tuned from 700 to $780 \mathrm{~nm}$. This work provides new insights into the strategy of designing fascinating TADF materials and represents an important step in the field of NIR OLED. ${ }^{95}$ 


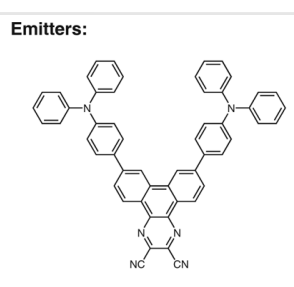

T1

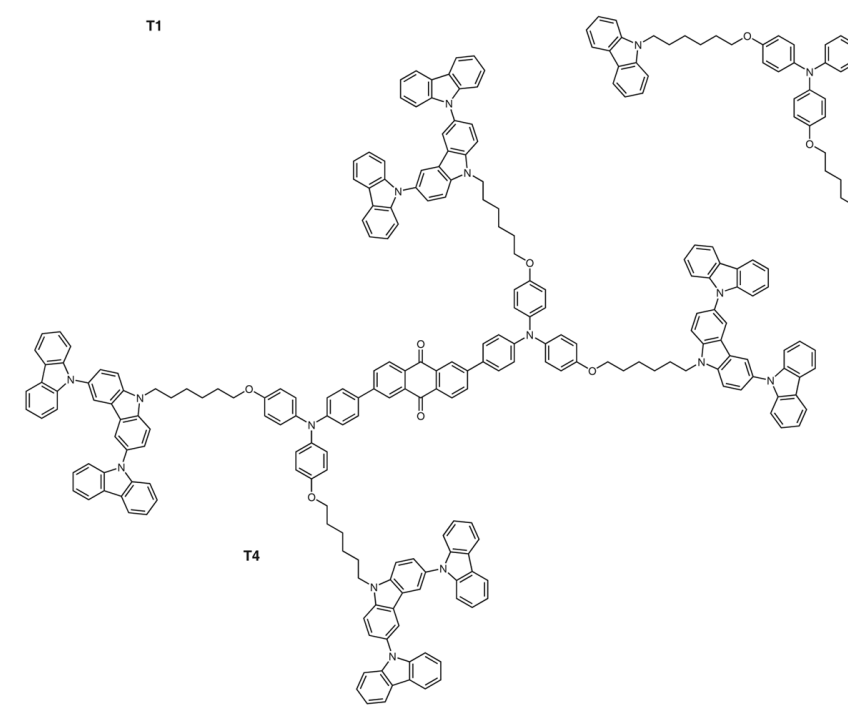

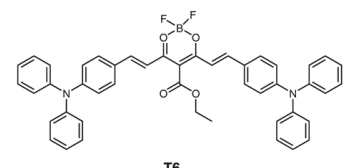

T6

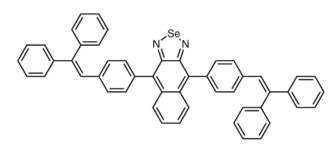

т9

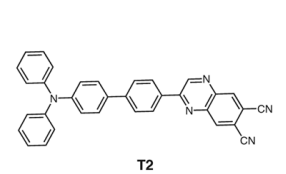

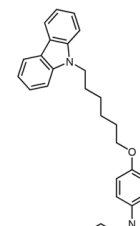

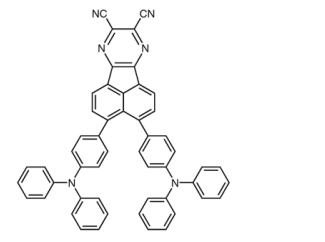

T5

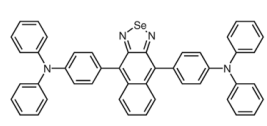

$\pi$

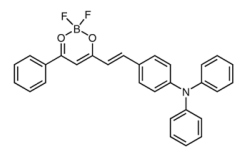

T8

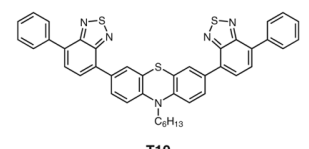

Hosts:

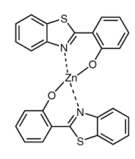

$\mathrm{Zn}(\mathrm{BTZ})_{2}$

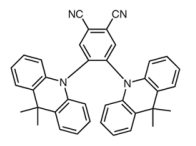

DMAC-PN

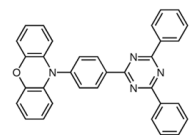

PXZ-TRZ

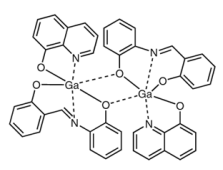

$\mathrm{Ga}_{2}(\mathrm{saph})_{2} \mathrm{q}_{2}$
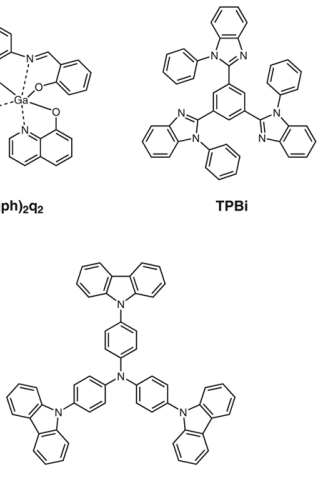

Figure 8 Chemical structures of unconventional NIR molecules and their hosts. 
It is difficult to achieve a small $\Delta E_{\mathrm{ST}}$ and a high radiative decay rate in a compound simultaneously by a common strategy of spatially separating the HOMO and LUMO, and thus TADF NIR emitters with PLQYare quite rare. In view of the abundant conventional fluorescent dopant (CFD) mentioned earlier, it is feasible to use TADF materials as hosts or sensitizers and CFDs as dopants to fabricate emitting layers in OLEDs. ${ }^{96-100}$ In this way, holes and electrons recombine in the host or sensitizer to form singlet and triplet excitons at a 1:3 ratio and then triplet excitons absorb the heat in the environment to form singlet excitons via a reversed intersystem crossing (RISC) due to the relatively small $\Delta E_{\mathrm{ST}}$. Radiative excitons are transferred to a fluorescent or phosphorescent dopant and then radiative decay for emitting photons through Förster resonance energy transfer (FRET, a nonradiative mechanism of energy transfer from a fluorophore in the excited state to a fluorophore in the ground state via dipole-dipole coupling) in PL or a charge carrier trapping mechanism in EL. In 2014, Adachi et al. used TADF molecules as assistant dopants that efficiently transfer all the electrically singlet and triplet excitons resonantly to the fluorescent emitters with different emission peaks to fabricate a series of OLEDs for blue, green, yellow, and red light emission. The performance, with EQEs as high as $13.4-18 \%$ for different colors, was good and demonstrated that in this way we can extend the emission of OLEDs to the NIR region. ${ }^{97}$ In 2019, Duan et al. fabricated a series of OLEDs to manipulate the interplay of host and TADF sensitizer to maximize the device performance. Adopting the TADF sensitization strategy, Qiao et al. fabricated OLEDs based on the TADF host DMAC-PN doped with T7 (Figure 8), whose molecular structure consists of 1,2-bis(9,9-dimethyl-9,10-dihydroacridine)-4,5-dicyanobenzene (DMAC-PN in Figure 8) and 4,9-bis(4-(diphenylamino)phenyl)-naphtho[2,3-c,1,2,5]selenadiazole (T7), exhibiting a maximum EQE of $2.65 \%$ with an EL peak at $730 \mathrm{~nm}$ and a small roll-off. In this work, the authors found that dopants with lateral bulky substituents could suppress the Dexter energy transfer (a short-range nonradiative process via collisional or exchange energy transfer) so that more singlet excitons could decay radiatively in the dopant. Therefore, the small roll-off can be ascribed to a relatively low concentration of triplet excitons, which may be quenched by TTA in the emitting layer. ${ }^{100}$ Meanwhile, Adachi et al. demonstrated that NIR electrophosphorescence up to $1100 \mathrm{~nm}$ can be achieved by using the TADF molecule 2phenoxazine-4,6-diphenyl-1,3,5-triazine (PXZ-TRZ) as the host matrix and as a triplet sensitizer to provide better balance between holes and electrons and a higher radiative exciton ratio in NIR-electrophosphorescence OLEDs as a result of the balanced bipolar charge-transport properties of PXZTRZ (Figure 8). The balanced carrier-transport properties were attributed to the spatially separated HOMO and LUMO distributions in PXZ-TRZ. Although optimized OLEDs based on blends of PXZ-TRZ as the TADF host and copper phthalocyanine (CuPc) or phthalocyanine (PtPc) as the phosphorescent emitter featured rather low EQE values of less than $0.1 \%$, limited by the relatively low phosphorescence efficiency of CuPc and PtPc, Adachi et al. believe that the issue will be addressed by using high-efficiency NIR emitters and a TADF-sensitized electrophosphorescence system. ${ }^{97}$

\section{TF (TTA) NIR-Emitting Materials}

Triplets fusion, also called TTA, is an alternative strategy to effectively exploit triplet excitons, and OLEDs based on this mechanism may achieve an $\eta_{\mathrm{r}}(\max )$ value of $62.5 \%$ because theoretically, $3 / 4$ triplet excitons and $1 / 4$ singlet excitons are generated by electricity and then two triplet excitons combine to form one singlet exciton. ${ }^{80}$ In 2016, Qiao et al. reported a high-performance NIR OLED based on TF, reaching a maximum EQE of $2.1 \%$ at $700 \mathrm{~nm}$ and maintaining a high EQE of about $2 \%$ in a wide range of current densities. The device was fabricated with a NIR emitter, 4,9-bis(4-(2,2-diphenylvinyl)phenyl)-naphtho[2,3c,1,2,5]selenadiazole (T9 in Figure 8), with a large LUMO/HOMO overlap and ambipolar character acting as a dopant in a bipolar host $\mathbf{G a}_{\mathbf{2}}(\mathbf{s a p h})_{\mathbf{2}} \mathbf{q}_{\mathbf{2}}$ (Figure 8). In this system, recombination of electrons and holes as well as TF occur in the host material and the singlet excitons are transferred to T9 via a FRET mechanism for radiative decay. ${ }^{82}$ Qiao et al. offer us a new device design strategy and molecular structure for NIR emission.

\section{NIR-Emitting Materials with Doublet Emission}

In 2015, Li et al. proposed a new open-shell molecule as an emitter in OLEDs, in which only one electron is populated in the highest singly occupied molecular orbital (SOMO) and can be excited to the lowest singly unoccupied molecular orbital (SUMO). Theoretically, the transition between SOMO and SUMO is spin-allowed, so the upper limit of IQE in the OLEDs is $100 \%$. Photons are generated by $D_{1}-D_{0}$ transitions in these neutral radicals; in this way, the poor exciton utilization efficiency of OLEDs can be solved. ${ }^{83}$ Later in 2018, Li et al. made a breakthrough in the NIR OLED field by fabricating high-performance OLEDs emitting at $710 \mathrm{~nm}$ (TTM-3NCz; Figure 7) and $703 \mathrm{~nm}$ with maximum EQEs of $27 \%$ and $17 \%$, respectively. ${ }^{84}$ This work demonstrated that open-shell doublet donor-radical molecules are promising candidate materials for high-efficiency OLED fabrication.

\section{NIR-Emitting Materials with "Hot-Exciton Mechanism"}

Sometimes, molecules with a certain structure may leverage excitons in higher excited states, such as the thirdexcited-triplet excitons $\left(T_{3}\right)$ for light emission in EL; this is also called the "hot-exciton" mechanism. In 2014, Yao et al. reported on the butterfly-shaped A-D-A type chromophore 
T10 (Figure 8), which exhibited a strong NIR fluorescence that peaked at $700 \mathrm{~nm}$ in film and the corresponding quantum efficiency reached $16 \%$. Remarkably, the NIR OLED based on $\mathbf{T 1 0}$ features a maximum EQE of $1.54 \%$ and a high radiative exciton ratio of $48 \%$ with quite a low efficiency rolloff. At a high current density of $300 \mathrm{~mA} \mathrm{~cm}^{-2}$, the EQE of the
S11-based device remained as high as $1.17 \%$. These authors established that a HLCT state exists for T10 in a low-polarity solvent or in the thin-film state and the high radiative single exciton ratio can be ascribed to the facile $T_{3}$ to $S_{1}$ RISC process in the HLCT state demonstrated by the similar natural transition orbitals of $\mathrm{S}_{1}$ and $\mathrm{T}_{3}$ states. Given the large

Table 2 Summary of chemical structures of NIR phosphorescent organometallic materials and their corresponding EQE and EL peaks

\begin{tabular}{|c|c|c|c|c|c|}
\hline Emitter & Host & EL peak $(n m)$ & Doping ratio (wt\%) & EQE (\%) & Ref. \\
\hline M1 & PVK & 1540 & 80 & - & 104 \\
\hline M2 & F8BT & 890 & & 62 & \\
\hline $\mathrm{M} 3(\mathbf{L n}=\mathbf{N d})$ & & 890,1070 & & 110 & \\
\hline $\mathrm{M} 3(\mathrm{Ln}=\mathrm{Er})$ & & 1540 & & 110 & \\
\hline $\mathrm{M} 3(\mathbf{L n}=\mathrm{Yb})$ & & 985 & & 110 & \\
\hline $\mathrm{M} 4(\operatorname{Ln}=\mathrm{Yb})$ & PPP-OR1 & 977 & & 0.001 & 111 \\
\hline$M 4(\operatorname{Ln}=\mathrm{Er})$ & MEH-PPV & 1560 & & 111 & \\
\hline M5 & $\mathrm{Alq}_{3}$ & 772 & & 8.5 & 25 \\
\hline M5 & $\mathrm{Alq}_{3}$ & 772 & 6 & 6.3 & 22 \\
\hline M6 & PVK:PBD & 896 & 4 & 3.8 & 24 \\
\hline M6 & PVK:PBD & 898 & 2 & 0.75 & 21 \\
\hline M6 & $\mathrm{Alq}_{3}$ & 900 & 4 & 3.8 & 21 \\
\hline M7 & $\mathrm{Alq}_{3}$ & 773 & 4 & 9.2 & 21 \\
\hline M8 & PVK:PBD & 774 & 2 & 3.0 & 21 \\
\hline M8 & PVK:PBD & 1005 & 4 & 0.20 & 21 \\
\hline M9 & $\mathrm{Alq}_{3}$ & 848 & 4 & 2.8 & 23 \\
\hline M10 & $\mathrm{Alq}_{3}$ & 846 & 4 & 1.5 & 23 \\
\hline M11 & & 760 & & 0.03 & 126 \\
\hline M12 & & 705 & & 10.5 & 127 \\
\hline M13 & & 700 & & 14.5 & 128 \\
\hline M13 & & 715 & & 10.5 & 127 \\
\hline M14 & & 720 & & 8.5 & 127 \\
\hline M15 & & 800 & & 1.2 & 125 \\
\hline M16 & PVK & 720 & 5 & 0.1 & 129 \\
\hline M18 & M17 & 620 & 0.3 & 9.2 & 131 \\
\hline M19 & PVK:PBD & 788 & 20 & 0.5 & 124 \\
\hline M20 & PVK:PBD & 791 & 20 & 0.5 & 124 \\
\hline M22 & DIC-TRZ & 760 & & 4.5 & 132 \\
\hline M23 & PVK:OXD7 & 709 & 5 & 1.28 & 121 \\
\hline M24 & PVK:OXD7 & 714 & 5 & 3.07 & 121 \\
\hline M25 & PVK:OXD7 & 714 & 5 & 2.44 & 121 \\
\hline M26 & PVK:PBD & 690 & 20 & 0.67 & 123 \\
\hline M27 & PVK:PBD & 753 & 20 & 0.3 & 123 \\
\hline M28 & $\mathrm{Ga}_{2}(\mathrm{saph})_{2} \mathrm{q}_{2}$ & 780 & 20 & 2.2 & 120 \\
\hline M29 & $\mathrm{Alq}_{3}$ & 718 & & 2.7 & 133 \\
\hline м30 & $\mathrm{Alq}_{3}$ & 814 & 6 & 1.5 & 133 \\
\hline M31:M32 & BP4mPy & 770 & & 0.067 & 134 \\
\hline$\left[\mathrm{Pt}(\mathrm{fprpz})_{2}\right]$ & & 740 & & 24 & 118 \\
\hline
\end{tabular}


dipole moment of the CT state and the certain degree of orbital overlap of the LE state, S11 displayed strong NIR emission and AIE behavior in the aggregated state. Despite D-A-D fluorophores having been widely studied, A-D-Atype molecules have been scarcely used because of the difficult monofunctionalization of the units in terms of synthesis. $^{70}$

\section{NIR Organometallic Phosphorescent Materials}

With regard to phosphorescent OLEDs, organometallic phosphorescent materials have several advantages over traditional fluorescent materials. First, because phosphorescence originates from triplet excitons' radiative decay, phosphorescent OLEDs can harvest both singlet and triplet excitons for light emission, so the ideal IQE may attain $100 \%$. Second, phosphorescence shows a longer decay lifetime from microsecond to millisecond. Third, the heavy metal ions in organometallic phosphorescent materials can facilitate the spin-orbit coupling effect, so the intersystem-crossing rates will increase and the phosphorescence radiative transitions from excited triplet to ground states will dominate in exciton deactivation pathways. The emissive mechanisms of metal complexes is complicated and the possible luminous mechanisms of metal complex materials consist of triplet metal-to-ligand charge transfer $\left({ }^{3} \mathrm{MLCT}\right)$, intraligand charge transfer $\left({ }^{3} \mathrm{ILCT}\right)$, ligand-toligand charge transfer $\left({ }^{3} \mathrm{LLCT}\right)$, ligand-to-metal charge transfer $\left({ }^{3} \mathrm{LMCT}\right)$, metal-metal-to-ligand charge transfer $\left({ }^{3} \mathrm{MMLCT}\right)$, and metal-to-ligand-ligand charge transfer $\left({ }^{3}\right.$ MLLCT $)$. The impact factors of excited states include the metal centers, chemical structures, and triplet-state energy levels of the ligands, local environment, and intermolecular interaction, so the photophysical properties of metal complexes such as absorption, emission wavelength, phosphorescence lifetime, and quantum efficiency can be modulated by varying the ligand structures and metal ions. ${ }^{101}$ In the following section, we summarize the phosphorescent NIR materials and classify them by central metals under appropriate optimization (Table 2).

\section{Lanthanide-Based Materials}

In general, organic dyes usually possess broad emission spectra, which is disadvantageous for the color purity of devices. On the contrary, lanthanide metal ions exhibit extremely sharp emission bands because their $4 \mathrm{f}$ orbitals are effectively shielded by the external forces of overlying $5 s^{2}$ and $5 p^{6}$ orbitals. For this reason, the emission and absorption bands ( $\mathrm{f}-\mathrm{f}$ transition) of metal ions become extremely sharp when electronic transitions occur from one
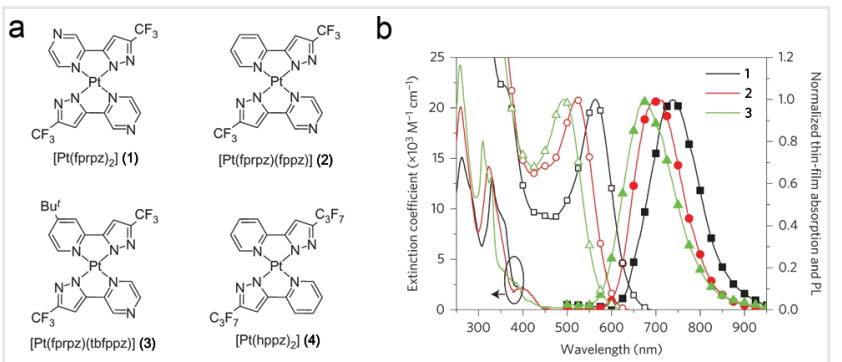

Figure 9 (a) Chemical structures of Pt(II) complexes 1-4. (b) Absorption spectra of 1-3 in THF (left, solid lines) and corresponding absorption spectra (right, unified symbols) and PL spectra (right, filled symbols) of 1-3 in solid film normalized at the peak wavelength are also shown. Reprinted with permission from Ref. 118. Copyright 2016 Springer Nature.

$\mathrm{J}$ state of an $\mathrm{f}^{\mathrm{n}}$ configuration to another $\mathrm{J}$ state of the same configuration, and the metal ions are generally excited by intramolecular energy transfer from the triplet states of a $\pi$ conjugated ligand in lanthanide organometallic complexes. Therefore, the color purity of lanthanide-based OLEDs can be very high. ${ }^{102}$ Complexes incorporating lanthanide materials like $\operatorname{Er}(\mathrm{III}), \mathrm{Yb}(\mathrm{III})$, and $\mathrm{Nd}(\mathrm{III})$ have attracted considerable attention due to their NIR emission. ${ }^{103}$

However, the luminescence efficiencies of lanthanide complexes also suffer from the "energy-gap law" and the low absorption coefficient of parity-forbidden $\mathrm{f}-\mathrm{f}$ transitions. ${ }^{104}$ To enhance the luminescence efficiencies of lanthanide complexes, several approaches have been employed, such as constituting a host-guest system in the emitting layer, changing the ITO or silicon-based substrates, and copolymerizing the organic ligands with a polymer main chain. ${ }^{62,104-107}$ In 1999 , the first phosphorescent OLEDs incorporating tris(8-hydroquinoline)erbium (III) (ErQ) as the neat emitting layer with an EL peak at 1.54 $\mu \mathrm{m}$ were fabricated by Curry et al., ${ }^{108,109}$ and were based on ITO or a silicon substrate. The room-temperature EL at 1.54 $\mu \mathrm{m}$ was ascribed to the intra-atomic transitions between the ${ }^{4} \mathrm{I}_{13 / 2}$ to ${ }^{4} \mathrm{I}_{15 / 2}$ levels in the $\mathrm{Er}^{3+}$ ion but at that time the authors were unable to establish the exact luminescence efficiencies for the OLEDs. Furthermore, by blending the lanthanide complex into a polymer matrix as the emitting layer, NIR OLEDs with higher efficiency were fabricated. The most common host polymers are MEH-PPV, PVK, and F8BT, whose emission peaks are 550, 450, and $550 \mathrm{~nm}$, respectively. ${ }^{59,105,106,110}$

In 2000, Sun et al. reported OLEDs using tris(acetylacetonato)(1,10-phenanthroline)erbium (M1 in Figure 10) doped into PVK with a doping ratio of $80 \mathrm{wt} \%$ as the emitting layer, exhibiting a NIR EL emission peaking at 1.54 $\mu \mathrm{m} .{ }^{104}$ In 2001, Slooff et al. reported an 890-nm luminescence from neodymium-doped PLEDs based on blends of F8BT and a lissamine-functionalized terphenyl-based neodymium complex (M2 in Figure 10) as the emitting layer. In 
Emitters:

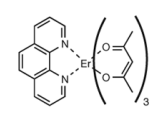

M1

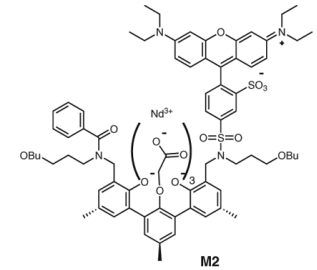

M2

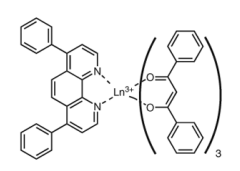

$\stackrel{M 3}{L n=N d, Y b, E r, E u}$

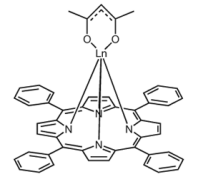

M4

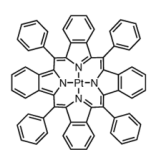

M5

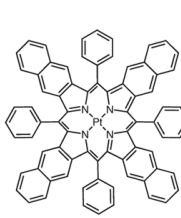

M6

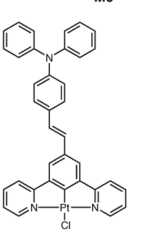

M15

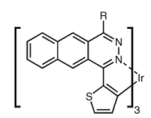

M21: $R=\prod^{s}$

M22: $\mathrm{R}=\underset{\mathrm{F}_{3} \mathrm{C}}{\mathrm{CF}_{3}}$

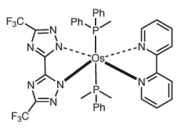

M31

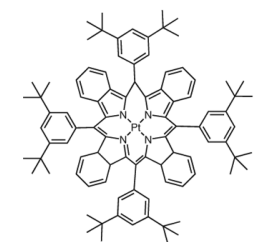

M7

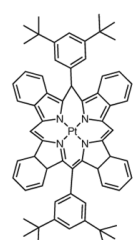

м8

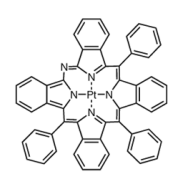

M9

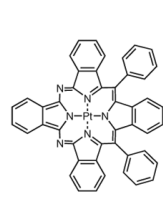

M10

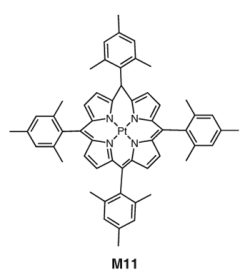

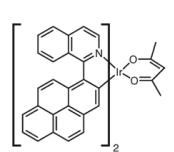

M16

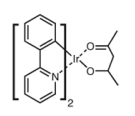

M17<smiles>c1ccc2c(c1)C1CCC23c2ccccc2C13</smiles>

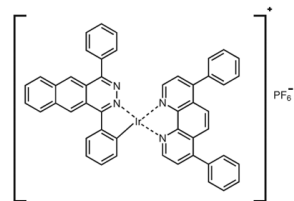

M19

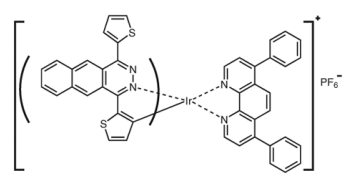

M20

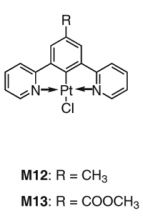
M13: $\mathrm{R}=\mathrm{COOCH}_{3}$ M14: $R=H$

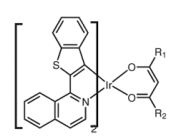

M23: $\mathrm{R}_{1}=\mathrm{CF}_{3}, \mathrm{R}_{2}=$ 年》

M24: $\mathrm{R}_{1}=\mathrm{R}_{2}=\mathrm{C}\left(\mathrm{CH}_{3}\right)_{3}$

M25: $R_{1}=R_{2}=$ IS
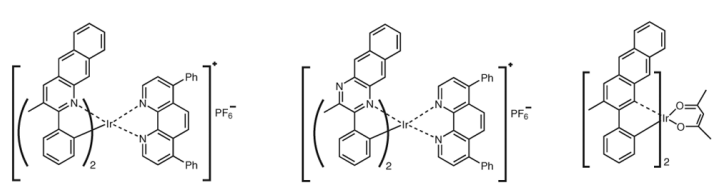

M28

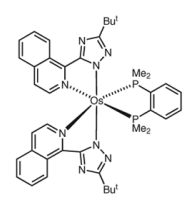

M29

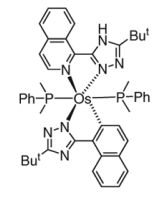

м30

Hosts:

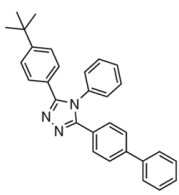

TAZ

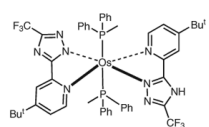

M32

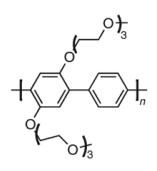

PPP-OR11

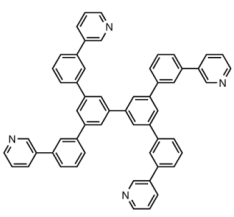

BP4mPy

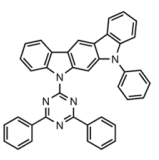

DIC-TRZ

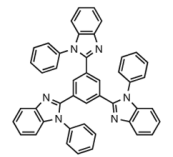

TPBi

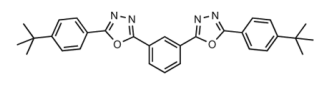

OXD7

Figure 10 Chemical structures of organometallic NIR phosphorescent materials and their hosts.

this work, lissamine acted as a sensitizer to transfer charges to $\mathrm{Nd}^{3+}$ followed by direct formation of triplets on the metal ions. ${ }^{62}$ In the same year, Kawamura et al. reported a series of OLEDs based on a tris(dibenzoylmethanato)(monobathophenanthroline)lanthanide(III) complex (lanthanide $=\mathrm{Nd}$, $\mathrm{Er}, \mathrm{Eu}$, and $\mathrm{Yb}, \mathbf{M} 3$ in Figure 10), displaying the EL emission bands ranging from 900 to $1600 \mathrm{~nm}$ at room temperature with EQEs below $0.03 \%{ }^{110}$ Harrison and coworkers fabri- cated NIR PLEDs using blends of MEH-PPV or bis-alkoxysubstituted poly( $p$-phenylene) (PPP-OR11 in Figure 10) as host materials and Yb-TPP-based or Er-TPP-based complexes as emitters (M4 in Figure 10, TPP $=5,10,15,20$ tetraphenylporphyrin). The Yb-based devices utilizing MEH-PPV or PPP-OR11 as hosts exhibited an EL peak at $977 \mathrm{~nm}$ and Er-based devices utilizing MEH-PPV as the host exhibited an EL peak at $1560 \mathrm{~nm}$. The visible part of EL was 
strongly quenched in all devices and in the PPP-OR11-based devices, the effect become significant due not only to a good spectral overlap between the Q-absorption bands of TPPs and MEH-PPV fluorescence but also to the better spectral overlap between the fluorescence of PPP-OR11 and the Soret absorption band of TPPs, which facilitate the FRET process. Interestingly, in the EL process, TPPs acted as sensitizers to transfer triplet excitons to the lanthanide ions followed by the phosphorescence radiative recombination in the metal ions. The Yb-based device using PPP-OR1 as the host showed a maximum EQE of $0.001 \%$ at $7 \mathrm{~V}$, which is five times higher than that of a Yb-based device using MEH-PPV as the host. ${ }^{111}$

\section{Transition Metal-Based Materials}

Transition metal complexes with strong phosphorescence emission have attracted intensive attention in recent years, thanks to their intriguing photophysical properties, especially for $\operatorname{Ir}(\mathrm{III})$ and $\mathrm{Pt}(\mathrm{II})$ complexes. ${ }^{108-110,112-117}$ The square-planar platinum complexes have a rigid molecular configuration, which could suppress nonradiative decay. However, the concomitant aggregation-induced quenching effect and relatively long phosphorescence lifetime may also lead to severe efficiency roll-off at a high current density in devices. Remarkably, the extremely high EQE record of $24 \%$ at $740 \mathrm{~nm}$ among NIR OLEDs was set by homoleptic Pt(II) complexes. ${ }^{118}$ Compared to the square-planar Pt(II) complexes, octahedral Ir(III) complexes have relatively short phosphorescence lifetimes, so they exhibit less efficiency roll-off in devices. ${ }^{111,118-124}$ The maximum EQEs of $3.4 \%$ at $702 \mathrm{~nm},{ }^{121} 3.1 \%$ at $714 \mathrm{~nm},{ }^{122}$ and $2.2 \%$ in the $750-800 \mathrm{~nm}$ range have been achieved by $\operatorname{Ir}($ III) complex-based devices. $^{111}$

\section{Pt-Based Phosphorescent Materials}

Since the first phosphorescent OLED based on Ptporphyrin complex (PtOEP) doped into $\mathbf{A l q}_{3}$ was reported by Baldo et al., ${ }^{113}$ Pt-porphyrin complexes have drawn extensive interest in recent years due to the significant absorption and emission in the red-to-NIR region. $^{21-25,119,125}$ As described before, the lone pairs on nitrogen atoms can coordinate to various metals and the porphyrin ring can be functionalized by a variety of groups; thus, rich and fascinating photophysical properties can be found in porphyrin-based materials. In the previous section, we have discussed porphyrin-based fluorescent materials, so in this subsection we will focus on Pt-porphyrin complexes for phosphorescence emission.

In 2007, Sun et al. studied the photophysical properties of several Pt-porphyrin derivatives in depth and fabricated devices using $\mathbf{A l q}_{3}$ or $\mathbf{C B P}$ as the host matrix doped with $\mathrm{Pt}$
(II)-tetraphenyltetrabenzoporphyrin (M5 in Figure 10) as a phosphor, and dimethyl-4,7-diphenyl-1,10-phenanthroline (BCP) as an electron-blocking layer, which exhibited a maximum EQE of $8.5 \%$ with the EL peaking at $772 \mathrm{~nm}$ and good color purity. ${ }^{25}$ Performances of the device exceeded those reported previously in works based on M5 doped in $\mathbf{A l q}_{\mathbf{3}}$ (EQE was $6.3 \%$ at $765 \mathrm{~nm}$ ). ${ }^{22}$ In a different approach to that of others, these authors fabricated the OLEDs with an emitting layer of CBP doped with 4 wt\% M5 and 2 wt\% PQIr (Ir(III) bis(2-phenylquinolyl- $N, \mathrm{C}^{2 \prime}$ )acetylacetonate) simultaneously, to suppress TTA and the aggregation of M5. Interestingly, they discovered that the field-induced charge pair dissociation mechanism dominates in the efficiency roll-off process rather than the TTA mechanism, from which they inferred that co-doping induced efficient cascade energy transfer from CBP through PQIr to M5. Therefore, the accumulation of high-density triplets in the emitting layer can be avoided. The co-doped OLED showed a maximum EQE of $8.5 \%$ with a less pronounced efficiency roll-off. ${ }^{25}$

To extend the NIR emission above $800 \mathrm{~nm}$, Sommer et al. used a blend of Pt(tptnp) as the dopant (tptnp: tetraphenyltetranaphtho[2,3]porphyrin, $\mathbf{M 6}$ in Figure 10) and PVK/PBD (6:4) as the host as an emitting layer to fabricate PLEDs with a strategy of extending conjugation in the porphyrin ligand. The devices based on the spin-coated emitting layer showed an EL peak at $896 \mathrm{~nm}$, and a maximum EQE of $0.4 \%$. Moreover, the optimized devices incorporating blends of $\mathbf{M 6}$ and CBP as the emitting layer, which were prepared by vapor deposition, featured a maximum EQE of $2.8 \%$ at $896 \mathrm{~nm}^{24}$ In 2011, with the similar strategy, these authors synthesized a family of $\pi$ extended Pt(II) porphyrins to fabricate a series of solutionprocessed PLEDs and vapor-deposited multilayer OLEDs with EL peaks ranging from 771 to $1005 \mathrm{~nm}$. The optimized PLED and OLED based on $\pi$-extended Pt porphyrins (M7 and M8 in Figure 10) exhibited EQEs of 9.2\% at $773 \mathrm{~nm}$ and 3.0\% at $774 \mathrm{~nm}$, respectively at that time. ${ }^{21}$ In 2016, Huang et al. reported vacuum-evaporated NIR OLEDs based on two Pt(II) azatetrabenzoporphyrin complexes (M9 and $\mathbf{M 1 0}$ in Figure 10), which featured the maximum EQE of $2.8 \%$ at $848 \mathrm{~nm}$ and $1.5 \%$ at $846 \mathrm{~nm}$, respectively. ${ }^{23}$

In the previous section we mentioned that copolymerizing the porphyrin rings with the polymer host can be an alternative method of achieving fluorescence emission. ${ }^{17,18,27}$ Regarding phosphorescent materials, in 2015, Freeman et al. copolymerized a dimesityl diphenyl porphyrin platinum (M11 in Figure 10) into a PFO host main chain connected by phenyl groups. By varying the loading of M11 $(0.5 \%, 1 \%, 2 \%$, and $5 \%)$, the PLEDs incorporating these copolymers as a neat emitting layer showed EL peaks at 665, 736 , and $818 \mathrm{~nm}$ with a shoulder peak at $642 \mathrm{~nm}$ and EQEs ranging from $0.17 \%$ to $0.48 \%{ }^{26}$ In 2016 , the same group reported a PLED incorporating M11 copolymerized with poly(diphenylanthracene) as an emitting layer. The device 
with $10 \%$ porphyrin loading showed an EL peak at $760 \mathrm{~nm}$ and an EQE up to $0.03 \%$, revealing a remarkable redshift compared to its PL peak at $666 \mathrm{~nm}$. They attributed the redshift phenomenon to the increased aggregation of the novel polymer backbone, which induced either polymerporphyrin or porphyrin-porphyrin aggregation. ${ }^{126}$

In addition to Pt porphyrins, the other Pt complexes may exhibit NIR emission by appropriate molecular design. In 2007, Cocchi and coworkers successfully fabricated OLEDs based on a series of terdentate cyclometalated phosphorescent Pt(II) complexes (M12-M14 in Figure 10) as emitters. ${ }^{127}$ Thanks to the high excimer emission quantum yields and the confined recombination zone in the complexes, the OLEDs emitting at 705 and $720 \mathrm{~nm}$ displayed excellent performances with EQEs up to $10.5 \% .{ }^{127}$ In 2008 , the same group reported an extremely efficient NIR OLED with an EL peak at $\sim 700 \mathrm{~nm}$ and a very high EQE of $14.5 \%$. The authors used the same series of terdentate cyclometalated efficient phosphorescent $\mathrm{Pt}(\mathrm{II})$ complexes (M12-M14) as pristine emitting layers to fabricate high-performance excimerbased NIR OLEDs. They demonstrated that inserting a $\mathrm{PbO}_{2}$ buffer layer between the $\mathrm{Ca}$ cathode and the electrontransporting layer can improves the device performance owing to the changed band bending between the electrontransport layer and the cathode. ${ }^{128}$ In 2013, Nisic and coworkers synthesized two kinds of Pt complexes, and the OLED based on the latter one (M31) showed a maximum EQE of $1.2 \%$ at $800 \mathrm{~nm} .{ }^{125}$ Moreover, several high-efficiency NIR OLEDs that emit light at $740 \mathrm{~nm}$ with a maximum EQE of $24 \pm 1 \%$ and a radiance of $(3.6 \pm 0.2) \times 10^{5} \mathrm{~mW} \mathrm{sr}^{-1} \mathrm{~m}^{-2}$ were reported by Tuong Ly et al. in 2017. It was demonstrated by them through XRD and theoretical calculations that the high performances can be ascribed to the high PLQY (about 81\%) and a superior edge-on orientation of the $\mathrm{Pt}(\mathrm{II})$ complex $\left[\mathrm{Pt}(\mathrm{fprpz})_{2}\right]$ in solid states. ${ }^{118}$ In this work, the authors used three kinds of 2pyrazinylpyrazolate $\mathrm{Pt}(\mathrm{II})$ phosphor complexes: [Pt(fprpz $)_{2}$ ], [Pt(fprpz)(fppz)], and [Pt(fprpz)(tbfppz)] (Figure 9), which were characterized by rather high PLQYs of $81 \%$ at $740 \mathrm{~nm}$, $55 \%$ at $703 \mathrm{~nm}$, and $82 \%$ at $673 \mathrm{~nm}$, respectively, in the thinfilm state, to fabricate OLEDs. To gain in-depth insights into the transition process, the authors employed wide-angle $\mathrm{X}$ ray scattering, angle-dependent luminescence, and timedependent DFT to determine the self-assembly and MMLCT properties at the molecular level. They demonstrated that in the solid state, the aggregation property of $\mathrm{Pt}(\mathrm{II})$ complexes is through $\pi$-interaction in the $\mathrm{d}_{\mathrm{z}} 2$ direction, and the MMLCT property along the Pt-Pt linkage seems to be an exciton-like model. Thanks to the short packing distance, the interaction among $5 \mathrm{~d}_{\mathrm{z}} 2$ (HOMO) in $\mathrm{Pt}(\mathrm{II})$ and $\pi^{*}$ orbitals (LUMO) in the ligands is strong. As a result, the excitons featuring a long diffusion length can be prevented from exciton-vibrational coupling and the nonradiative deactivation process will be suppressed. ${ }^{118}$

\section{Ir-Based Materials}

The OLED featuring exclusive NIR emission was reported by Williams et al. in 2006, ${ }^{129}$ which exhibited a maximum EQE of nearly $0.25 \%$ at $720 \mathrm{~nm}$, including blends of iridium (III) bis(1-pyrenyl-isoquinolinato- $N, C$ )acetylacetonate (M16 in Figure 10) and polymer hosts PVK and PBD. Furthermore, the optimized devices with an extra hole-blocking layer of BCP and an electron-injecting layer $\mathbf{A l q}$ showed a higher performance but less NIR purity, which can be attributed to emission from exciplex formation among BCP, PVK, and PBD. ${ }^{129}$ Wong and coworkers synthesized a family of borylated Ir(III) cyclometalated complexes with oligothiophene-based ligands to fabricate OLEDs. The EL spectra's relative intensities in the NIR region are significantly higher than those in PL spectra. Although the performances of devices needed to be improved, it is believed that by modulating the ligands, the $\operatorname{Ir}(\mathrm{III})$ complexes with an electron-withdrawing borylated substituent would show greater potential in NIR emission. ${ }^{130}$

In 2007, Tsuzuki and coworkers fabricated OLEDs using a host/guest system of M17 and M18 (Figure 10) [where M17 is $\operatorname{bis}\left(2-\right.$ phenylpyridinato- $N, C^{2 \prime}$ )iridium(acetylacetonate) and $\mathbf{M 1 8}$ is tris(1-phenylisoquinolinolato- $\left.C^{2}, N\right)$ iridium (III)] because of the relatively larger bandgap, higher $T_{1}$ state energy, and good hole-transport property of M17. The green phosphorescence of M17 was effectively quenched by the $1 \mathrm{wt} \% \mathbf{M 1 8}$, suggesting that the triplet energy of M17 was efficiently transferred to M18. The maximum EQE of the devices was 9.2\% with an EL peak at $620 \mathrm{~nm}$ at $0.3 \mathrm{wt} \% \mathbf{M 1 8}$, and the driving voltage was $4.4 \mathrm{~V}$ at a luminance of $100 \mathrm{~cd}$ $\mathrm{m}^{-2} \cdot{ }^{131}$ Furthermore, the extension of $\pi$-conjugated systems and decoration of the ligands have been demonstrated as effective ways to extend the emission into the NIR region and maintain a rigid molecular configuration concomitantly although it may bring difficult problems in synthesis and purification, which can be attributed to the increased steric hindrance and decreased solubility of the materials. ${ }^{62}$ In 2015, Xin et al. designed two NIR-emitting cationic iridium (III) complexes M19 and M20 (Figure 10) and the OLEDs based on them displayed a constant EQE of around $0.5 \%$ over a large current density range of $1-100 \mathrm{~mA} \mathrm{~cm}{ }^{-2} .{ }^{124}$ With the same synthetic strategy, in 2017, Xue et al. reported two novel NIR-emitting homoleptic facial Ir(III) complexes based on extended $\pi$-conjugated benzo[g]phthalazine ligands, tris [1,4-di(thiophen-2-yl)benzo[g]phthalazine]iridium(III)

(M21 in Figure 10) and tris[1-(2,4-bis(trifluoromethyl) phenyl)-4-(thiophen-2-yl)-benzo[g]phthalazine] iridium (III) (M22 in Figure 10), whose PLQYs in solution are 5.2\% at $824 \mathrm{~nm}$ and $17.3 \%$ at $765 \mathrm{~nm}$, respectively. The authors fabricated NIR OLEDs using 2,4-diphenyl-6-bis(12-phenylindolo[2,3-a]-carbazole-11-yl)-1,3,5-triazine (DIC-TRZ in Figure 10) as the host matrix doped with two $\operatorname{Ir}(\mathrm{III})$ complexes, featuring an ultrapure EL emission at $760 \mathrm{~nm}$ and a maximum EQE of $4.5 \%$. EQEs of these devices remain 
as high as $3.5 \%$ at $100 \mathrm{~mA} \mathrm{~cm}^{-2}$. This work may provide us with new insights into the fabrication of high-efficiency and small roll-off NIR OLEDs. ${ }^{132}$ Similarly, by introducing the electron-rich thiophene groups in ligands, Kesarkar et al. reported three cyclometalated Ir(III) complexes doped in PVK and OXD7 (Figure 10) with different $\beta$-diketonate ancillary ligands with increasing conjugation, which exhibited efficient NIR emission, namely M23, M24, and M25 (Figure 10). The device based on $\mathbf{M 2 4}$ showed a remarkable EQE of 3.07\% with an EL peak at $714 \mathrm{~nm} .{ }^{121}$ In 2012, two NIR cationic iridium (III) complexes M26 and M27 were synthesized and characterized by Rao and coworkers. The use of $\mathrm{sp}^{2}$-hybridized $\mathrm{N}$ opposite the chelating $\mathrm{N}$ atom in the ligand of $\mathbf{M 2 7}$ caused the emission to redshift $60-80 \mathrm{~nm}$ as compared to M26. ${ }^{123}$ In 2013, these authors changed part of the ligands in M27 and synthesized a new cationic complex M28 with a strong NIR emission. The device based on M28 as an emitter and the bipolar host $\mathbf{G a}_{\mathbf{2}}(\mathbf{s a p h})_{\mathbf{2}} \mathbf{q}_{\mathbf{2}}$ exhibited a maximum EQE of $2.2 \%$ and a constant EQE of around $2 \%$ over a wide range of current densities from 3 to $100 \mathrm{~mA} \mathrm{~cm}^{-2} .{ }^{120}$ These works indicate that Ir-based materials hold great promise for the usage in NIR OLEDs in terms of small efficiency roll-off.

\section{Other Transition-Metal Materials}

In 2009, Lee at el. developed a series of charge-neutral Os (II) isoquinolyl triazolate complexes (M29 and M30 in Figure 10) with trans- and cis-configurations to fabricate a series of devices based on $\mathbf{A l q} \mathbf{q}_{3}, \mathbf{T P B i}$, and TAZ (Figure 10) as hosts. It is noteworthy that by using $\mathbf{A l q}_{\mathbf{3}}$ as the host, a device with EL peaking at $814 \mathrm{~nm}$ and an EQE of $1.5 \%$ is achieved, and a device with EL peaking at $718 \mathrm{~nm}$ and an EQE of $2.7 \%$ is achieved, indicating that the Os(II) complexes with lower intrinsic excited states, short radiative lifetime, and nonionic natures are promising in NIR OLEDs. ${ }^{133}$ In 2014, by employing a highly conjugated isoquinolinyl azolate chelate as ligands to decrease the excited-state energy level, Liao and coworkers reported a NIR OLED based on $\mathrm{Os}(\mathrm{bpftz})_{2}\left(\mathrm{PPh}_{2} \mathrm{Me}\right)_{2}$ and $\left[\mathrm{Os}(\mathrm{bpy})(\mathrm{dttz})\left(\mathrm{PPhMe}_{2}\right)_{2}\right]$ (M31 and M32 in Figure 10) doped in BP4mPy (Figure 10) emitting at $770 \mathrm{~nm}$ with an EQE of $0.067 \%{ }^{134}$

\section{NIR QD Materials}

QD nanocrystals (QDs) display readily tunable luminescence throughout the whole NIR region due to their large Bohr exciton radius ( $20 \mathrm{~nm}$ for PbS and 46 for PbSe). Unlike typical organic materials, QDs are not subject to strong vibrational coupling between the ground and excited states, which causes broadened spectra and increased nonradiative decay rates. Moreover, QDs also feature a higher luminance efficiency, higher photostability, and narrower spectral emissions than organic materials. The PL and EL emission wavelengths can be tuned by varying the QD size according to the quantum confine effect, so QDs outperform organic materials at wavelengths beyond $1000 \mathrm{~nm}$ and are promising candidates for NIR light-emitting materials. ${ }^{135-146}$ However, because the surface states on QDs can quench excitons, their surface structures should be carefully processed. In addition, the interdot carrier transport can lead to unfavorable transport-assisted trapping, which results in self-quenching in QDs. Passivation with a widebandgap inorganic or organic shell, capping with insulating organic ligands, and incorporation into a host matrix are the established methods applied to improve device performance. These methods, however, may bring some problems such as low current density, charge injection imbalance, and exciton ionization induced by large bias voltages. In this section, typical NIR QLEDs with emitting layers that were treated by different passivation methods will be discussed. ${ }^{136,142}$ There is difficulty in the synthesis of monodispersed QDs, which might increase the cost of QLEDs. ${ }^{147}$

The earliest NIR QLED was reported by Banin et al. in 2008, who utilized core-shell InAs-ZnSe QDs embedded in MEH-PPV to fabricate QLEDs with EQE values of about $0.5 \%$ and EL peaks varying from 1 to $1.3 \mu \mathrm{m}$. In this work, they demonstrated that the optimized core-shell nanocrystal structure shields the excitons localized in the core from the host deactivation path and maintains the energy transfer from the host through a Förster or Dexter mechanism. ${ }^{135}$ In the same year, a highly efficient hybrid organic-inorganic nanocrystal NIR QLED based on PbS nanocrystals that emit at $1.2 \mu \mathrm{m}$ with a maximum EQE of $1.15 \%$ was reported by Bourdakos et al., ${ }^{142}$ outperforming the previously reported nanocrystal-based device. ${ }^{135}$ The excitons are directly created on the oleic acid-capped PbS nanocrystals, thus removing the requirement of efficient energy transfer from organic molecules to nanocrystals. A solution-processed hybrid polymer-nanocrystal NIR LED based on PbSe nanocrystals embedded in a conjugated polymer MEH-PPV that emits at $1280 \mathrm{~nm}$ with an EQE of $0.83 \%$ was reported by Choudhury et al. in 2009. In this work, the PbSe nanocrystals acted as carrier traps where the injected electrons and holes recombine to form excitons for radiative recombination. They demonstrated that increased carrier trapping leads to more efficient exciton formation on the emitting sites of PbSe nanocrystals, resulting in a higher quantum efficiency. ${ }^{144}$

In addition to IV-VI semiconductor nanocrystals such as $\mathrm{PbS}, \mathrm{PbSe}$, indirect-bandgap-semiconductor Si nanocrystals can be used in NIR QLEDs. In 2011, Cheng et al. obtained highly efficient Si nanocrystal LEDs with EQEs of 8.6\% and $6.7 \%$ at emission wavelengths of 853 and $777 \mathrm{~nm}$, respectively. To our knowledge, this is the best performance among nanocrystal-based LEDs emitting at similar wavelengths. The high device performance can be ascribed to the high 
intrinsic PLQYs of $45 \%$ and $43 \%$ at the peak wavelengths of 853 and $777 \mathrm{~nm}$ for $\mathrm{Si}$ nanocrystals of 5- and 3-nm diameter, respectively, that were chemically passivated with ligands of 1-dodecene in a uniformly dispersed solution. This work demonstrated that nanocrystals of indirect-gap $\mathrm{Si}$ semiconductors have exceptional photophysical properties in the NIR region. ${ }^{137}$

By using different organic ligands for surface passivation, the interdot distance of QDs can be tuned and their performance may be improved. In 2012, Hu et al. fabricated QLEDs that showed a maximum EQE of $0.73 \%$ and an EL peak at $1412 \mathrm{~nm}$ by using $\mathrm{ZnO}$ nanocrystals as the electrontransporting layers and a ligand replacement technique to activate PbSe QDs. After treatment with 1,2-ethanedithiol (EDT), the absorption peaks and PL spectrum of the PbSe QD film exhibited significant redshifts due to QD film densification. ${ }^{146}$ Moreover, by tuning the distance between adjacent $\mathrm{PbS}$ QDs and varying their size, Sun et al. fabricated a series of thin-film QLEDs with EL emission peaks ranging from 884 to $1618 \mathrm{~nm}$, and the best one showed a peak EQE of $2.0 \%$ at $1054 \mathrm{~nm}$ with a maximum radiance of $6.4 \mathrm{~W} \mathrm{sr}^{-1}$ $\mathrm{m}^{-2}$. In this work, the distance between adjacent $\mathrm{PbS}$ dots is modulated by varying the lengths of the mercapto alkyl carboxylic acids from 3-mercaptopropionic acid, 6-mercaptohexanoic acid, and 8-mercaptooctanoic acid (MOA) to 11-mercaptoundecanoic acid, which acts as bifunctional linker molecules. It was proven that a small interdot distance in the QD layer facilitates efficient charge transport and charge injection as well as leads to undesired exciton dissociation. By contrast, a larger interdot distance promotes exciton radiative recombination, but charge injection becomes difficult and the screening of the external field is weakened, which may cause exciton ionization. Considering all the factors, the authors found that the optimum charge injection balance and radiative exciton recombination were achieved by using MOA as linker molecules. ${ }^{138}$ Similarly, in 2012, Ma et al. reported a new way of producing highquality $\mathrm{PbS}$ nanocrystalline films acting as both electrontransporting and emitting layers in NIR LEDs by the benzenedithiol (BDT) cross-linking method, which exhibits a $20 \%$ quantum yield in solution. The BDT-treated PbS nanocrystal films have lower doping concentration, lower conductivity, and less free-carrier density than the conventional EDT-treated nanocrystal films. Therefore, the undesirable nonradiative Auger recombination, which is proportional to the cube of the carrier density, can be suppressed in the BDT-treated films. Such devices exhibit a maximum EQE of $0.72 \%$ at $1050 \mathrm{~nm} .{ }^{140}$

Furthermore, properly processed QDs can be used as electron-transporting or hole-transporting layers. Yang et al. reported on NIR LEDs based on QD materials as the hole-transporting, electron-transporting, and light-emitting layers, which are the so-called "all-quantum-dot IR LEDs" (Figure 11). By tuning the particle sizes, the QLEDs
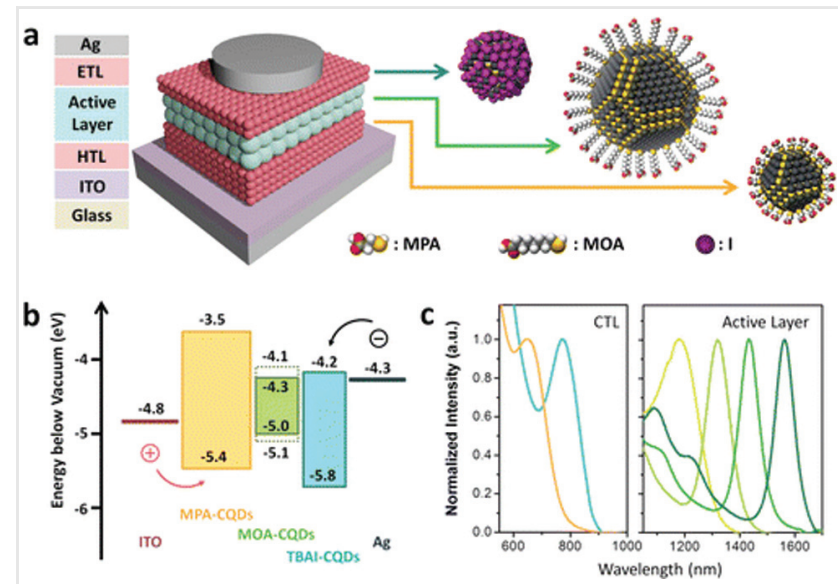

Figure 11 (a) Schematic illustration of the device architecture. Arrows represent the layers consisting of CQDs with different sizes and ligand passivation. (b) QLED energy diagram. The solid line in the active layer represents the CQDs emitting at $1622 \mathrm{~nm}$ and the dashed line represents the CQDs emitting at $1220 \mathrm{~nm}$. (c) CQD absorption spectra acting as charge-transporting layers. (labs $=1178,1320,1427$, and 1572 nm). Reprinted with permission from Ref. 141. Copyright 2015 American Chemical Society.

emit from 1220 to $1622 \mathrm{~nm}$ and the best one, emitting at $1350 \mathrm{~nm}$, achieved a maximum EQE of $1.58 \%$ with a low $V_{\text {on }}$ of $1.2 \mathrm{~V}$. Because of the low mobility of colloidal QDs (CQDs), the injected carriers accumulated in the active layer mostly for radiative or Auger recombination rather than the SRH recombination. The simplified material requirements and solution-processed methods provide us with a new way to fabricate cost-effective NIR LEDs. ${ }^{141}$ In 2015, Yan et al. used a blue GaN LED as the excitation source and PbSe QDs as the NIR luminescent material to fabricate NIR LEDs with a maximum EQE of $2.52 \%$ at $950 \mathrm{~nm}$. Moreover, EQE values of $1.83 \%$ and $0.67 \%$ were also achieved for 1550 and $1960 \mathrm{~nm}$ QLEDs by changing the active-layer thickness. ${ }^{139}$

As described above, the use of core-shell QDs can serve as a useful way to inhibit surface oxidation and nonradiative pathways in QLEDs. ${ }^{135}$ In 2015, Supran et al. reported that LEDs based on PbS-CdS core-shell QDs showed a peak EQE of $4.3 \%$ with a $V_{\text {on }}$ as low as $0.6 \mathrm{~V}$ and EL peaks ranging from 1163 to $1341 \mathrm{~nm}$; the performance of which significantly surpassed those of devices based on PbS core-only QDs (Figure 12). It was shown that the CdS shell synthesized by cation exchange significantly mitigates the in situ nonradiative pathways in PbS QDs, such as multicarrier Auger recombination, exciton energy transfer to conductive charge-transporting layers (CTLs), and exciton dissociation at CTL/QD interfaces; thus, the device performances can be enhanced significantly. ${ }^{142}$

Like OLEDs or PLEDs, by embedding QDs into a suitable matrix, we can obtain high-performance QLEDs due to the 

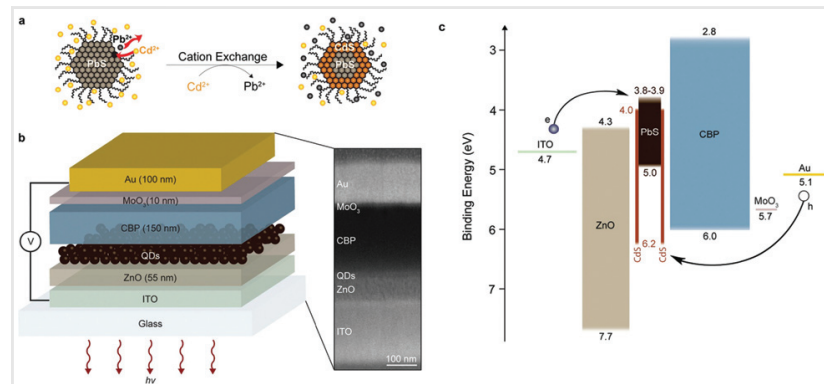

Figure 12 (a) Schematic illustration of the cation-exchange reaction used to convert core-only PbS QDs into core-shell PbS--CdS QDs. (b) Device architecture (left) and cross-sectional SEM image (right) of the QLED based on these QDs. (c) The QLED's flat-band energy level diagram. The electron affinity of $\mathrm{PbS}$ is tuned from approximately 3.8 to $3.9 \mathrm{eV}$ by the reduction in core size (from 4.0 to $3.6 \mathrm{~nm}$ ) accompanying cation exchange. Reprinted with permission from Ref. 142. Copyright 2015 Wiley-VCH.

decreased interdot quenching. In 2016, Bansal et al. reported on highly luminescent CdS QDs, which featured a comparatively high PLQY of $69 \%$ in solution and $34 \%$ in a neat thin film. They were embedded in an organic semiconductor matrix to fabricate a NIR solution-processed hybrid LED. The QLED based on a blend of QDs with a CBP matrix, with a volume ratio of 0.05:0.95, shows a maximum EQE of $0.62 \%$ at a peak emission wavelength of $760 \mathrm{~nm}$. For the CBP host QLEDs, the hole injection barrier is as high as $0.45 \mathrm{eV}$; thus, the EQE is much lower than might be expected for the high PLQY of CdS QDs, and the devices need to be optimized further. ${ }^{136}$ According to previous reports, CQD films that have high luminescence efficiency and high carrier mobility simultaneously cannot be obtained, because high-mobility materials also feature a high exciton dissociation rate. ${ }^{142}$ Nevertheless, Gong et al. addressed this issue with a strategy of embedding nanocrystals in a hybrid perovskite matrix with high carrier mobility. With the benefit of the excellent carrier-transport property and the suitable perovskite matrix consisting of a certain I/Br ratio, the transport-assisted trapping losses such as SRH recombination and the self-quenching on the CQD films can be inhibited, so the device emitting at $1391 \mathrm{~nm}$ achieves a maximum EQE of $5.2 \%$ and a record high power conversion efficiency (defined as the ratio of the energy of a photon emitted to the energy of an electron injected) of $4.9 \%$ so far with emission beyond $900 \mathrm{~nm} .{ }^{145}$

Considering the size-dependent emission of QDs and their large exciton Bohr radius of commonly used $\mathrm{Pb}$ chalcogenide, it is vital to find an effective way of synthesizing monodisperse QDs for QLED fabrication. As described above, researchers have applied suitable coreshell structures or organic ligands to passivate the surface defects and suppress interdot quenching. Through the modulation of organic ligands on the surface of QDs, they have successfully tuned the interdot spacing and synthesized high-efficiency and high-uniformity QD films for NIR emission (Table 3). Therefore, it is reasonable to assume a bright future for QDs used in NIR QLED.

Table 3 Summary of NIR QD and perovskite materials and their corresponding EQE and EL peaks

\begin{tabular}{|c|c|c|c|}
\hline Materials & EL Peak (nm) & EQE (\%) & Ref. \\
\hline $\mathrm{MAPbl}_{3} \mathrm{QDs}$ & 748 & 10.4 & 161 \\
\hline $\mathrm{CdS} \mathrm{QDs}^{\mathrm{a}}$ & 760 & 0.62 & 136 \\
\hline $\mathrm{PEA}_{2}(\mathrm{MA})_{n-1} \mathrm{~Pb}_{n} \mathrm{I}_{3 n+1} \mathrm{QWs}$ & 760 & 8.8 & 163 \\
\hline $\mathrm{NMA}_{2}(\mathrm{FA})_{n-1} \mathrm{~Pb}_{n}(1 / \mathrm{Br})_{3 n+1} \mathrm{QWs}$ & 763 & 11.7 & 151 \\
\hline Si QDs (3 nm) & 777 & 6.7 & 137 \\
\hline Bulk FAPbl $_{3}$ & 803 & 20.7 & 150 \\
\hline Si QDs (5 nm) & 853 & 8.6 & 137 \\
\hline FPMAI-MAPb ${ }_{0.6} \mathrm{Sn}_{0.4} \mathrm{I}_{2} \mathrm{Br}$ & 854 & 1.4 & 147 \\
\hline FPMAI-MAPb ${ }_{0.6} \mathrm{Sn}_{0.4} \mathrm{I}_{2.2} \mathrm{Br}_{0.8}$ & 871 & 1.8 & 147 \\
\hline FPMAI-MAPb ${ }_{0.8} \mathrm{Sn}_{0.2} \mathrm{I}_{3}$ & 877 & 0.7 & 147 \\
\hline \multirow[t]{5}{*}{ PbS QDs } & 884 & 0.43 & 138 \\
\hline & 1054 & 2.0 & \\
\hline & 1232 & 0.45 & \\
\hline & 1432 & 0.035 & \\
\hline & 1618 & 0.035 & \\
\hline FPMAI-MAPb ${ }_{0.6} \mathrm{Sn}_{0.4} \mathrm{I}_{2.4} \mathrm{Br}_{0.6}$ & 885 & 1.7 & 147 \\
\hline FPMAI-MAPb ${ }_{0.6} \mathrm{Sn}_{0.4} \mathrm{I}_{2.6} \mathrm{Br}_{0.4}$ & 900 & 2.6 & 147 \\
\hline FPMAI-MASnl 3 & 901 & 0.9 & 147 \\
\hline FPMAI-MAPb ${ }_{0.6} \mathrm{Sn}_{0.4} \mathrm{I}_{2.8} \mathrm{Br}_{0.2}$ & 909 & 3.3 & 147 \\
\hline FPMAI-MAPb ${ }_{0.6} \mathrm{Sn}_{0.4} \mathrm{I}_{3}$ & 917 & 5 & 147 \\
\hline FPMAI-MAPb ${ }_{0.4} \mathrm{Sn}_{0.6} \mathrm{l}_{3}$ & 928 & 2.7 & 147 \\
\hline FPMAI-MAPb ${ }_{0.2} \mathrm{Sn}_{0.8} \mathrm{l}_{3}$ & 943 & 0.6 & 47 \\
\hline $\mathrm{MASnl}_{3}$ & 945 & 0.72 & 156 \\
\hline $\mathrm{CsSnl}_{3}$ & 950 & 3.8 & 55 \\
\hline \multirow[t]{3}{*}{$\mathrm{PbSe}$} & 950 & 2.52 & 139 \\
\hline & 1550 & 1.83 & \\
\hline & 1950 & 0.67 & \\
\hline PbS (BDT-treated) & 1050 & 0.72 & 140 \\
\hline \multirow[t]{4}{*}{ PbS CQDs } & 1220 & 0.79 & 141 \\
\hline & 1350 & 1.58 & \\
\hline & 1450 & 0.37 & \\
\hline & 1622 & 0.14 & \\
\hline PbS-CdS QDs & 1230 & 4.3 & 142 \\
\hline $\mathrm{PbS}$ & 1250 & 1.15 & 143 \\
\hline $\mathrm{PbSe}^{\mathrm{b}}$ & 1280 & 0.83 & 144 \\
\hline PbS QDs & 1391 & 4.7 & 145 \\
\hline PbSe (EDT-treated) & 1412 & 0.73 & 146 \\
\hline
\end{tabular}

aEmitters doped in CBP host material.

bemitters doped in MEH-PPV (6 wt\%) host material. 


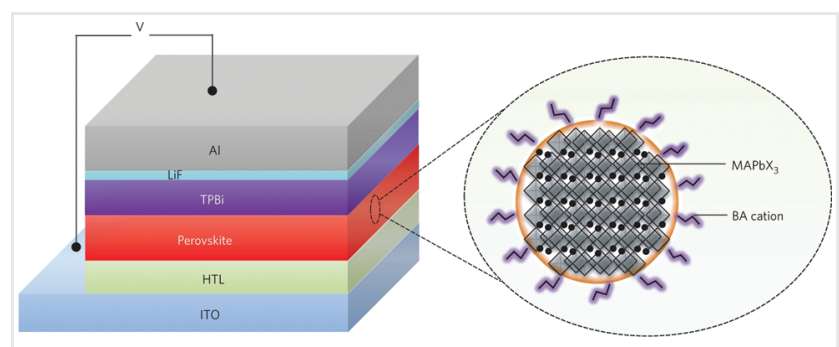

Figure 13 Device structure of PeLED. Inset: schematic representation of a nanometer-sized grain with $n$-butylammonium cations passivating their surfaces. Reprinted with permission from Ref. 161. Copyright 2016 Springer Nature.

\section{NIR Perovskite Materials}

Considering the "energy-gap law" for organic materials and the difficulty in synthesizing monodispersed QDs, it is very important to investigate alternative NIR-emitting materials. Organometal halide perovskites with a high PLQY, excellent carrier mobility, tunable bandgap, long exciton diffusion length, solution processability, and good color purity are promising light-emitting materials for LED fabrication. In recent years, organometal halide perovskites have been found to possess excellent EL properties ranging from the visible to the NIR range. However, nonradiative recombination caused by defects and leakage current on the surface limits their EL performance. These issues have been addressed in numerous studies. ${ }^{148-151}$

Typical organometal halide perovskites can be described with the general formula of $\mathrm{L}_{2}(\mathrm{SMX})_{n-1} \mathrm{MX}_{4}$, where $\mathrm{M}$ is a divalent metal cation, $\mathrm{X}$ is a halide, $\mathrm{L}$ and $\mathrm{S}$ are organic cations with long and short chains, respectively, and $n$ is the number of semiconducting $\mathrm{MX}_{4}$ monolayer sheets within the two organic insulating layers. When $n=\infty$, the structure corresponds to the 3D perovskite $\mathrm{SMX}_{3}$; when $n=0$, the structure corresponds to the $2 \mathrm{D}$ perovskite $\mathrm{L}_{2} \mathrm{MX}_{4}$; and $n$ values in between are quasi-2D perovskites (Figure 14c). Hybrid 3D perovskites feature high PLQYs, high carrier mobility, and small exciton binding energy; thus, the 3D perovskite materials can achieve high PLQY at high-excitation photon fluences where radiative bimolecular recombination dominates. The 2D perovskites generally exhibit good film-formation properties, a larger exciton-binding energy than 3D perovskites, and low PLQYs at room temperature due to the fast exciton-quenching rates. Nevertheless, the quasi-2D layered perovskites (also called a "Ruddlesden-Popper structure") with a self-organized quantum well (QW) structure show exceptional optical properties when used in PeLEDs. ${ }^{151}$

\section{D Perovskite Materials}

In 2015, Gil-Escrig et al. reported on PeLEDs based on $\mathrm{CH}_{3} \mathrm{NH}_{3} \mathrm{PbI}_{3}$ exhibiting a peak EQE of $0.04 \%$ at an emission

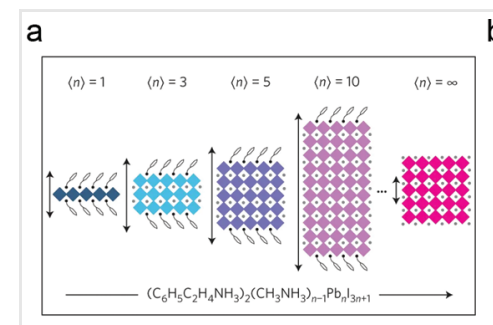

b
C

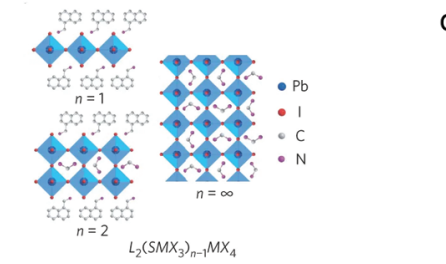

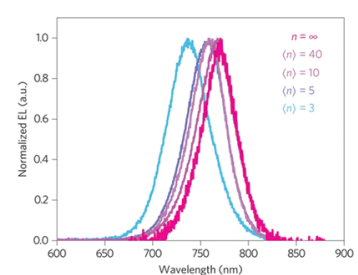

d

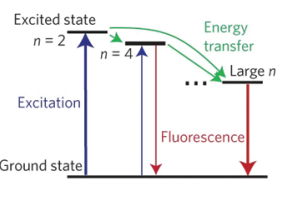

Figure 14 (a) Unit cell structures of $\mathrm{PEA}_{2}\left(\mathrm{CH}_{3} \mathrm{NH}_{3}\right)_{n-1} \mathrm{~Pb}_{n} \mathrm{I}_{3 n+1}$ perovskites with different $(n)$ values from $2 \mathrm{D}(n=1)$ to $3 \mathrm{D}(n=\infty)$. (b) EL spectra of PeLEDs based on PEA $2\left(\mathrm{CH}_{3} \mathrm{NH}_{3}\right)_{n-1} \mathrm{~Pb}_{n} \mathrm{I}_{3 n+1}$ with different $<n>$ values. ${ }^{163}$ (c) Schematic representation of the structures of $(\mathrm{NMA})_{2}\left(\mathrm{FAPbl}_{3}\right)_{n-1} \mathrm{Pbl}_{4}$. (d) Schematic cascade energy transfer in MQWs. Excitation energy is transferred from small- $n$ QWs to large- $n$ QWs followed by the emission in the large- $n$ QWs. ${ }^{151}$ Reprinted with permission from Ref. 163. Copyright 2016 Springer Nature. Reprinted with permission from Ref. 151. Copyright 2016 Springer Nature.

peak of $765 \mathrm{~nm}$. Given the very low PLQY of the active layer, this work implied that the prospect for improvement in EL performance was bright for NIR PeLEDs. ${ }^{152}$ Later in 2015, Kumawat et al. studied the structural, electrical, and optical properties of four 3D perovskite semiconductors with the formula $\mathrm{CH}_{3} \mathrm{NH}_{3} \mathrm{PbX}_{3}$ (where $\mathrm{X}$ is a halide ion) with different bandgaps ranging from the visible to the NIR region by changing the halide ion, substrate wetting conditions, and crystallite size. A maximum EQE of $0.28 \%$ was achieved for a NIR device with an EL peak of approximately $760 \mathrm{~nm} .{ }^{153}$ In the same year, Wang et al. introduced the multifunctional interfacial layer of polyethyleneimine (PEI), which is conducive to forming highquality perovskite thin films with good crystallinity features, long PL lifetimes, and good surface coverage for the solution-processed PeLEDs. They concluded that an organic polyamine can be used to improve the interface between the oxide electron-transporting layer and the perovskite emissive layer to produce a low-defect and high-quality heterojunction to improve device performance. Consequently, these authors successfully fabricated solution-processed PeLEDs with an EL peak of $768 \mathrm{~nm}$ and a maximum EQE of $3.5 \%$ (average EQE is $2.2 \%$ ). ${ }^{154}$ Unlike OLEDs or QLEDs, the EQE of the NIR PeLEDs increases with the increase in current density because higher excitation densities facilitate radiative bimolecular recombination in PeLEDs instead of the exciton-exciton and exciton-charge quenching that occurred in OLEDs and QLEDs. 
To further effectively extract the light trapped in LEDs, namely to improve the light-outcoupling efficiency, many methods such as using low-index grids and buckling patterns have been investigated. However, these methods are always associated with complicated fabrication processes with the emission spectra and directionality distorted. ${ }^{154-159}$ Intriguingly, Cao et al. have exploited perovskites prepared by introducing amino-acid additives in the precursor solutions to fabricate efficient and bright NIR PeLEDs that can make full use of the light trapped in the devices because the spontaneously formed submicrometerscale structures significantly increase the outcoupling efficiency. Because of the amino-acid additives, the perovskite surface defects are passivated and nonradiative recombination can be reduced; thus, the maximum EQE of $20.7 \%$ at a current density of $18 \mathrm{~mA} \mathrm{~cm}{ }^{-2}$ was achieved for the PeLED emitting at $803 \mathrm{~nm} .{ }^{150}$ In 2018, Giuri et al. obtained high-efficiency NIR formamidinium lead iodide $\left(\mathrm{FAPbI}_{3}\right)$ PeLEDs by introducing starch to manipulate the nanostructure of perovskite films, which exhibits a high radiance up to $206.7 \mathrm{~W} \mathrm{sr}^{-1} \mathrm{~m}^{-2}$ at the very high current density of $1000 \mathrm{~mA} \mathrm{~cm}^{-2}$, EQEs of approximately $5 \%$, and EL wavelengths ranging from 751 to $801 \mathrm{~nm}$. The addition of starch helped the formation of an $\alpha-\delta$ mixed phase that can improve the radiative bimolecular recombination and circumvent losses at the interface between the emissive layer and the hole/electron-transporting layer; the improved substrate coverage of the perovskite film can inhibit current leakage and modulate the dielectric landscape, so that the light-outcoupling efficiency can be increased. ${ }^{160}$

Many reports have demonstrated that the photophysical properties of perovskite materials can be modulated by changing the halide composition. In 2017, Xiao et al. proposed that long-chain ammonium halides can act as surfactants that terminate the grain growth of $3 \mathrm{D}$ perovskite in the perovskite solutions during the film-forming process, producing small-grain-sized, ultrasmooth, pinhole-free, and compact perovskite films. After the addition of longchain ammonium halides, the 3D nanometer-sized perovskite grains reduce their dimensions and start a transition from 3D to quasi-2D-like layered perovskite structures. By varying the ratio of $\mathrm{BAX}: \mathrm{MAPbX}_{3}(\mathrm{BAX}=n$-butylammonium halides; $\mathrm{X}=\mathrm{Br}$ and $\mathrm{I} ; \mathrm{MA}=$ methylammonium) in perovskite precursor solutions and incorporating them in LEDs, they realized that the EQEs of 20:100 Br-PeLEDs emitting at $513 \mathrm{~nm}$ and 20:100 I-PeLEDs emitting at $748 \mathrm{~nm}$ reached $9.3 \%$ and $10.4 \%$, respectively (Figure 13 ). ${ }^{161}$ In 2019, Qiu et al. reported mixed $\mathrm{Pb}-\mathrm{Sn}$ halide perovskitebased NIR LEDs with tunable emission peaks from 850 to $950 \mathrm{~nm}$ by varying the $\mathrm{Pb}: \mathrm{Sn}$ ratio or introducing a bromide. With the addition of 4-fluorobenzylammonium iodide (FPMAI) additives in the perovskite precursor, they obtained ultrasmooth nanocrystalline perovskite films and fabricated a PeLED with a maximum EQE of $5.0 \%$, a low $V_{\text {on }}$ of $1.65 \mathrm{~V}$, and an emission peak at $917 \mathrm{~nm}$ by using $\mathrm{Pb}-\mathrm{Sn}$ mixed perovskite with a $\mathrm{Pb}: \mathrm{Sn}$ ratio of $3: 2 .{ }^{147}$

Considering the use of toxic elements such as $\mathrm{Cd}$ and $\mathrm{Pb}$ in QLEDs and PeLEDs, which raises environmental and health issues, Hong et al. reported on a lead-free NIR PeLED based on a low-temperature solution-processed $\mathrm{CsSnI}_{3}$ perovskite. They used two methods, namely, the one-pot solution synthesis and the toluene dripping method, to prepare the $\mathrm{CsSnI}_{3}$ films and discovered that the $\mathrm{CsSnI}_{3}$ films prepared by the latter method showed compact micrometer-sized $\mathrm{CsSnI}_{3}$ grains with few pinholes and cracks at the grain boundary. The NIR LEDs based on such high-quality $\mathrm{CsSnI}_{3}$ films achieved a maximum EQE of 3.8\% at $4.5 \mathrm{~V}$, a maximum radiance of $40 \mathrm{~W} \mathrm{sr}^{-1} \mathrm{~m}^{-2}$, and an EL peak at $950 \mathrm{~nm}$. The characterization showed that the $\mathrm{CsSnI}_{3}$ film made by toluene dripping exhibits a faster PL decay, lower nonradiative recombination rates, and lower trap density of states than the $\mathrm{CsSnI}_{3}$ film prepared by onepot solution synthesis, which explains the high performance of the former. ${ }^{155}$ Additionally, Tan et al. fabricated methylammonium tin iodide ( $\mathrm{MASnI}_{3}$ )-based PeLEDs with a peak EQE of $0.72 \%$ and an EL peak at $945 \mathrm{~nm}$ and achieved spectrum tunability by varying the $\mathrm{Br}$ content in these perovskite materials. ${ }^{156}$ In 2017, Zhao et al. reported a nanocrystal film preparation strategy of using 4-fluorophenylmethylammonium iodide (FPMAI) as additives in situ to confine the crystal growth of the perovskite and achieved a high-quality perovskite film. Because of the good perovskite film, NIR LEDs based on $\mathrm{CH}_{3} \mathrm{NH}_{3} \mathrm{PbI}_{3}$ emitting at $749 \mathrm{~nm}$ exhibited a maximum EQE of $7.9 \%, 40$ times higher than the one without additives. It can be concluded that the additives passivate the defects on the perovskite surface, thereby enhancing the LED performance. ${ }^{162}$

\section{Quasi-2D Layered Perovskite Materials}

In 2016, Yuan et al. investigated a series of perovskite mixed material based on $\left(\mathrm{C}_{8} \mathrm{H}_{9} \mathrm{NH}_{3}\right)_{2}\left(\mathrm{CH}_{3} \mathrm{NH}_{3}\right)_{n-1} \mathrm{~Pb}_{n} \mathrm{I}_{3 n+1}$ consisting of different quantum-size-tuned grains that transfer photogenerated excitons to the lowest-bandgap emitter in the mixture. In this work, they increased the number of $\mathrm{PbI}_{6}$ monolayers $\langle n>$ to make the average dimensionally tuned perovskite in the solid state for exploring how $<n>$ affects the luminescence property of quasi-2D and 3D perovskite materials (Figure 14a, b). In consequence, they found that the $\mathrm{EQE}$ and the radiance for $n=5$ reach up to $8.8 \%$ for $200-\mathrm{nm}$ films and $80 \mathrm{~W} \mathrm{sr}^{-1} \mathrm{~m}^{-2}$ respectively, with an EL peak at about $750 \mathrm{~nm}$. It was demonstrated that the fast PL decay at shorter wavelengths in these materials can be ascribed to the intraband carrier funneling process, and the relatively lower PLQY can be attributed to the dominance of trap-assisted nonradiative recombination. They suggested that there is an increased 
local exciton density in low-bandgap and large- $n$ grains from the energy funnel process, so the high PLQY under low excitation can be achieved effectively. ${ }^{163}$ Similarly, Wang et al. presented solution-processed PeLEDs based on selforganized multiple QWs (MQWs) with good film morphology. To make good MQW perovskite films, they dissolved a precursor solution of 1-naphthylmethylamine iodide (NMAI), formamidinium iodide (FAI), and $\mathrm{PbI}_{2}$ with a molar ratio of 2:1:2 in $\mathrm{N}, \mathrm{N}$-dimethylformamide (DMF) to deposit perovskite films (named $\mathrm{NFPI}_{7}$ ). The authors then used NMAI, formamidinium bromide (FABr), and $\mathrm{PbI}_{2}$ with a molar ratio of 2:1:2 in DMF to deposit new perovskite MQW films $\mathrm{NFPI}_{6} \mathrm{~B}$ with better crystallinity than $\mathrm{NFPI}_{7}$ because of the changed halide composition in the precursor solution. A peak EQE of $11.7 \%$ at $2.6 \mathrm{~V}$ with a current density of $38 \mathrm{~mA} \mathrm{~cm}^{-2}$ and an EL emission peak at $763 \mathrm{~nm}$ was achieved in the LEDs based on $\mathrm{NFPI}_{6} \mathrm{~B}$ (Figure $14 \mathrm{c}, \mathrm{d}$ ). In the MQW perovskite films, exciton decay is slower than the energy transfer. Most of the photogenerated excitons can be transferred from the small- $n$ QWs to the large- $n$ QWs, in which $n$ is the number of $\mathrm{MX}_{4}$ layers, so the local exciton density is high in the large- $n$ QWs, which results in radiative recombination. ${ }^{151}$

Researchers have exploited 3D, quasi-2D, and 2D hybrid perovskite materials to make full use of the advantages of these materials, such as high carrier mobility, high PLQY, and tunable spectra (Table 3). Although long-term instability remains an issue and health or environmental concerns still exist, efforts are being made to find appropriate materials and optimized devices for lowering the driving voltages and circumventing the health and environmental risks.

\section{Conclusions and Outlook}

In conclusion, many difficulties were encountered when discovering and designing high-efficiency NIR-emitting materials. For organic materials, the performance is seldom satisfactory in the long-wavelength region, according to the "energy-gap law" and the aggregation-induced quenching effect. Extending the emission range further into the NIR region in OLEDs requires careful management of both the aggregation situation and the nonradiative deactivation pathways of the excited states in active layers. ${ }^{9}$ Researchers carefully design the molecular structure of organic emitters and tune their energy levels to extend their emission to the NIR region through introducing different donor or acceptor moieties. By rigidifying the molecular backbone and by judicious molecule design, we can circumvent some negative factors to the greatest possible extent. The socalled "energy-gap law" is only applicable to organic molecules with similar structures, and we may thus find some exceptions and then summarize them to form a feasible molecule design strategy. ${ }^{9}$ Doping and copolymerizing emitters into hosts are established methods for the fabrication of emitting layers with the aim of suppressing the aggregation-induced quenching effect. In these LEDs, the emitters accept excitons from hosts, followed by the radiative recombination of excitons in the emitting center. To efficiently leverage all the excitons generated in OLEDs, researchers have synthesized materials with TADF, TTA, a doublet radical mechanism, and organometallic materials. The organometallic materials perform best in all kinds of organic materials from the perspective of the EQEs of LEDs with emission peaks near $700 \mathrm{~nm} .{ }^{118}$ As mentioned in the Introduction, the potential applications of NIR LEDs in general span from security to biomedicine (e.g., blood oximetry, photodynamic therapy instruments, night-vision display). In addition to some common NIR applications, lowtoxicity organic materials have a definite advantage over inorganic counterparts in the territory of in vivo applications such as bioelectronics, though the competition by inorganic NIR emitters is strong in terms of efficiency, radiance, and durability. Besides, the relatively low-cost organic materials have great potential in large-area fabrication due to their solution-processability and flexibility.

As for QD materials featuring high PLQY and brightness, small FWHM, and tunable emission, they still suffer from severe self-quenching problems in solid-state films because of the enhanced transport-assisted trapping caused by the coupling between QDs. ${ }^{146}$ Another issue is that the surfaces of QDs should be carefully processed because active surface states may quench radiative excitons. The spectral tunability originates from the quantum size effect of QDs, by which the bandgaps of QDs increase as their sizes decrease. Thus, QDs perform well in the wavelength range above $1000 \mathrm{~nm}$ when compared with organic materials and perovskite materials. Moreover, the spectrally narrow emission of QDs compared with organic and inorganic emitters makes QDs excellent luminescent sources. Thus, NIR QLEDs have the potential in military applications, such as night-vision-readable displays ${ }^{1}$ and iris identification systems, ${ }^{7}$ due to their narrow emission. Similarly, considering the longer penetration depths and lower damage to living tissues of NIR light, the NIR QLEDs with emission from 800 to $1700 \mathrm{~nm}$ have a competitive advantage in the field of biomedical imaging and optical diagnosis. ${ }^{6}$

Perovskite materials feature high color purity and quantum efficiencies, good color tunability, and high flexibility, but their exciton binding energy is low, so they exhibit severe exciton dissociation when used as emitting materials at room temperature. In addition, it is hard to fabricate high-stability perovskite devices because the PeLEDs are usually driven at a high voltage. Moreover, Most QD materials and perovskite materials contain $\mathrm{Pb}$ and $\mathrm{Cr}$, elements that are detrimental to human health and the 
environment, and this limits their future application in daily life and in vivo applications. By the careful choice of suitable additives in the precursor solutions and changing the content ratios of perovskite materials, researchers have successfully made high-quality thin films for PeLED fabrication and tuned the emission peaks of the devices further to the NIR region. Furthermore, the self-organized QW structure from the quasi-2D perovskites helps in understanding the structure-property relationships of perovskite materials and alternative candidates for NIR emission. The perovskite materials show best performances in terms of EQE when compared with QDs and organic materials. The high EQEs and radiances of PeLEDs also make perovskite materials very promising for night-vision display.

We hope that this review of NIR emitting materials for LED fabrication will stimulate further investigation in this area, at both industrial and academic levels. And further basic studies in the luminescence mechanism will promote the understanding of material design. All in all, the existing problems will be solved at some point, and undoubtedly, a bright future can be assumed for commercial applications of these NIR LEDs.

\section{Funding Information}

The authors thank the National Key R\&D Program of China (2019YFA0705900 and 2017YFA0204701) and the National Natural Science Foundation of China (21572234, 2166 1132006, and 91833304) for their financial support.

\section{References}

(1) Qi, J.; Qiao, W.; Wang, Z. Y. Chem. Rec. 2016, 16, 1531.

(2) Pansare, V.; Hejazi, S.; Faenza, W.; Prud'homme, R. K. Chem. Mater. 2012, 24, 812.

(3) Smith, A. M.; Mancini, M. C.; Nie, S. Nat. Nanotechnol. 2009, 4, 710.

(4) Le, S. T.; Kanesan, T.; Bausi, F.; Haigh, P. A.; Rajbhandari, S.; Ghassemlooy, Z.; Papakonstantinou, I.; Popoola, W. O.; Burton, A.; Le Minh, H.; Cacialli, F.; Ellis, A. D. Opt. Lett. 2014, 39, 3876.

(5) Haigh, P. A.; Bausi, F.; Ghassemlooy, Z.; Papakonstantinou, I.; Le Minh, H.; Fléchon, C.; Cacialli, F. Opt. Express 2014, 22, 2830.

(6) Maggini, L.; Cabrera, I.; Ruiz-Carretero, A.; Prasetyanto, E. A.; Robinet, E.; De Cola, L. Nanoscale 2016, 8, 7240.

(7) Kono, M.; Ueki, H.; Umemura, S. Appl. Opt. 2002, 41, 7429.

(8) Segal, M.; Baldo, M. A.; Holmes, R. J.; Forrest, S. R.; Soos, Z. G. Phys. Rev. B: Condens. Matter 2003, 68, 075211.

(9) Zampetti, A.; Minotto, A.; Cacialli, F. Adv. Funct. Mater. 2019, 29, 1807623.

(10) Gao, Q.; Wang, Z. Y. Chem. Asian J. 2010, 5, 1006.

(11) Dou, L.; Liu, Y.; Hong, Z.; Li, G.; Yang, Y. Chem. Rev. 2015, 115, 12633.

(12) Luo, J.; Xie, Z.; Lam, J. W. Y.; Cheng, L.; Tang, B. Z.; Chen, H.; Qiu, C.; Kwok, H. S.; Zhan, X.; Liu, Y.; Zhu, D. Chem. Commun. 2001, 18,1740 .
(13) Baldo, M. A.; O’Brien, D. F.; Thompson, M. E.; Forrest, S. R. Phys. Rev. B: Condens. Matter 1999, 60, 14422.

(14) Veldhuis, S. A.; Boix, P. P.; Yantara, N.; Li, M.; Sum, T. C.; Mathews, N.; Mhaisalkar, S. G. Adv. Mater. 2016, 28, 6804.

(15) Burroughes, J. H.; Bradley, D. D. C.; Brown, A. R.; Marks, R. N.; Mackay, K.; Friend, R. H.; Burns, P. L.; Holmes, A. B. Nature 1990, 347, 539.

(16) Baigent, D. R.; Hamer, P. J.; Friend, R. H.; Moratti, S. C.; Holmes, A. B. Synth. Met. 1995, 71, 2175.

(17) Iqbal, R.; Moratti, S. C.; Holmes, A. B.; Yahioglu, G.; Milgrom, L. R.; Cacialli, F.; Morgado, J.; Friend, R. H. J. Mater. Sci. - Mater. Electron. 2000, 11, 97.

(18) Morgado, J.; Cacialli, F.; Friend, R. H.; Iqbal, R.; Yahioglu, G.; Milgrom, L. R.; Moratti, S. C.; Holmes, A. B. Chem. Phys. Lett. 2000, 325, 552.

(19) Ostrowski, J. C.; Susumu, K.; Robinson, M. R.; Therien, M. J.; Bazan, G. C. Adv. Mater. 2003, 15, 1296.

(20) Fenwick, O.; Sprafke, J. K.; Binas, J.; Kondratuk, D. V.; Di Stasio, F.; Anderson, H. L.; Cacialli, F. Nano Lett. 2011, 11, 2451.

(21) Graham, J. P.; Mininni, P. D.; Pouquet, A.; Shelton, A. H.; Schanze, K. S.; Xue, J. Phys. Rev. E: Stat. Nonlinear Soft Matter Phys. 2011, 84, 016314.

(22) Borek, C.; Hanson, K.; Djurovich, P. I.; Thompson, M. E.; Aznavour, K.; Bau, R.; Sun, Y.; Forrest, S. R.; Brooks, J.; Michalski, L.; Brown, J. Angew. Chem. 2007, 119, 1127.

(23) Huang, L.; Park, C. D.; Fleetham, T.; Li, J. Appl. Phys. Lett. 2016 109, 233302.

(24) Sommer, J. R.; Farley, R. T.; Graham, K. R.; Yang, Y.; Reynolds, J. R.; Xue, J.; Schanze, K. S. ACS Appl. Mater. Interfaces 2009, 1 , 274.

(25) Sun, Y.; Borek, C.; Hanson, K.; Djurovich, P. I.; Thompson, M. E.; Brooks, J.; Brown, J. J.; Forrest, S. R. Appl. Phys. Lett. 2007, 90, 213503.

(26) Freeman, D. M. E.; Tregnago, G.; Rodriguez, S. A.; Fallon, K. J.; Cacialli, F.; Bronstein, H. J. Org. Semicond. 2015, 3, 1.

(27) Iqbal, R.; Yahioglu, G.; Milgrom, L.; Moratti, S. C.; Holmes, A. B.; Cacialli, F.; Morgado, J.; Friend, R. H. Synth. Met. 1999, 102, 1024.

(28) Stender, B.; Völker, S. F.; Lambert, C.; Pflaum, J. Adv. Mater. 2013, 25, 2943.

(29) Havinga, E. E.; ten Hoeve, W.; Wynberg, H. Polym. Bull. 1992, 29, 119.

(30) Brocks, G.; Tol, A. J. Phys. Chem. 1996, 100, 1838.

(31) Brocks, G.; Tol, A. Synth. Met. 1996, 76, 213.

(32) Ellinger, S.; Graham, K. R.; Shi, P.; Farley, R. T.; Steckler, T. T.; Brookins, R. N.; Taranekar, P.; Mei, J.; Padilha, L. A.; Ensley, T. R.; Hu, H.; Webster, S.; Hagan, D. J.; Van Stryland, E. W.; Schanze, K. S.; Reynolds, J. R. Chem. Mater. 2011, 23, 3805.

(33) Minotto, A.; Murto, P.; Genene, Z.; Zampetti, A.; Carnicella, G.; Mammo, W.; Andersson, M. R.; Wang, E.; Cacialli, F. Adv. Mater. 2018, 30, 1706584.

(34) Hendriks, K. H.; Li, W.; Wienk, M. M.; Janssen, R. A. J. Am. Chem. Soc. 2014, 136, 12130.

(35) Murto, P.; Minotto, A.; Zampetti, A.; Xu, X.; Andersson, M. R.; Cacialli, F.; Wang, E. Adv. Opt. Mater. 2016, 4, 2068.

(36) Parker, T. C.; Patel, D. G.; Moudgil, K.; Barlow, S.; Risko, C.; Brédas, J.-L.; Reynolds, J. R.; Marder, S. R. Mater. Horiz. 2015, 2, 22.

(37) Kronemeijer, A. J.; Gili, E.; Shahid, M.; Rivnay, J.; Salleo, A.; Heeney, M.; Sirringhaus, H. Adv. Mater. 2012, 24, 1558.

(38) Yuen, J. D.; Kumar, R.; Zakhidov, D.; Seifter, J.; Lim, B.; Heeger, A. J.; Wudl, F. Adv. Mater. 2011, 23, 3780. 
(39) Fenwick, O.; Fusco, S.; Baig, T. N.; Di Stasio, F.; Steckler, T. T.; Henriksson, P.; Fléchon, C.; Andersson, M. R.; Cacialli, F. APL Mater. 2013, 1, 032108.

(40) Shahid, M.; Ashraf, R. S.; Huang, Z.; Kronemeijer, A. J.; McCarthy-Ward, T.; McCulloch, I.; Durrant, J. R.; Sirringhaus, H.; Heeney, M. Chem. Sci. 2012, 3, 181.

(41) Pandey, L.; Risko, C.; Norton, J. E.; Brédas, J.-L. Macromolecules 2012, 45, 6405.

(42) Yang, R.; Tian, R.; Hou, Q.; Yang, W.; Cao, Y. Macromolecules 2003, 36, 7453.

(43) Yang, R.; Tian, R.; Yan, J.; Zhang, Y.; Yang, J.; Hou, Q.; Yang, W.; Zhang, C.; Cao, Y. Macromolecules 2005, 38, 244.

(44) Tregnago, G.; Steckler, T. T.; Fenwick, O.; Andersson, M. R.; Cacialli, F. J. Mater. Chem. C 2015, 3, 2792.

(45) Kronemeijer, A. J.; Gili, E.; Shahid, M.; Rivnay, J.; Salleo, A.; Heeney, M.; Sirringhaus, H. Adv. Mater. 2012, 24, 1558.

(46) Yang, J.; Jiang, C.; Zhang, Y.; Yang, R.; Yang, W.; Hou, Q.; Cao, Y. Macromolecules 2004, 37, 1211.

(47) Sharbati, M. T.; Panahi, F.; Shourvarzi, A.; Khademi, S.; Emami, F. Optik 2013, 124, 52.

(48) Sun, M.; Jiang, X.; Liu, W.; Zhu, T.; Huang, F.; Cao, Y. Synth. Met. 2012, 162, 1406.

(49) Sun, M.; Jiang, X.; Wang, L.; He, C.; Du, B.; Yang, R.; Cao, Y. J. Polym. Sci., Part A: Polym. Chem. 2008, 46, 3007.

(50) Qian, G.; Dai, B.; Luo, M.; Yu, D.; Zhan, J.; Zhang, Z.; Ma, D.; Wang, Z. Y. Chem. Mater. 2008, 20, 6208.

(51) Qian, G.; Zhong, Z.; Luo, M.; Yu, D.; Zhang, Z.; Wang, Z. Y.; Ma, D. Adv. Mater. 2009, 21, 111.

(52) Du, X.; Qi, J.; Zhang, Z.; Ma, D.; Wang, Z. Y. Chem. Mater. 2012, 24, 2178.

(53) Yang, Y.; Farley, R. T.; Steckler, T. T.; Eom, S.-H.; Reynolds, J. R.; Schanze, K. S.; Xue, J. Appl. Phys. Lett. 2008, 93, 163305.

(54) Chen, M.; Perzon, E.; Andersson, M. R.; Marcinkevicius, S.; Jönsson, S. K. M.; Fahlman, M.; Berggren, M. Appl. Phys. Lett. 2004, 84, 3570.

(55) Steckler, T. T.; Lee, M. J.; Chen, Z.; Fenwick, O.; Andersson, M. R.; Cacialli, F.; Sirringhaus, H. J. Mater. Chem. C 2014, 2, 5133.

(56) Nielsen, C. B.; Turbiez, M.; McCulloch, I.Adv. Mater. 2013,25, 1859.

(57) Zambounis, J. S.; Hao, Z.; Iqbal, A. Nature 1997, 388, 131.

(58) Bronstein, H.; Chen, Z.; Ashraf, R. S.; Zhang, W.; Du, J.; Durrant, J. R.; Shakya Tuladhar, P.; Song, K.; Watkins, S. E.; Geerts, Y.; Wienk, M. M.; Janssen, R. A. J.; Anthopoulos, T.; Sirringhaus, H.; Heeney, M. J. Am. Chem. Soc. 2011, 133, 3272.

(59) Stas, S.; Balandier,J.-Y.; Lemaur, V.; Fenwick, O.; Tregnago, G.; Quist, F.; Cacialli, F.; Cornil, J.; Geerts, Y. H. Dyes Pigm. 2013, 97, 198.

(60) Zhang, K.; Tieke, B. Macromolecules 2008, 41, 7287.

(61) Beyerlein, T.; Tieke, B.; Forero-Lenger, S.; Brütting, W. Synth. Met. 2002, 130, 115.

(62) Slooff, L. H.; Polman, A.; Cacialli, F.; Friend, R. H.; Hebbink, G. A.; van Veggel, F. C. J. M.; Reinhoudt, D. N. Appl. Phys. Lett. 2001, 78, 2122.

(63) Li, P.; Fenwick, O.; Yilmaz, S.; Breusov, D.; Caruana, D. J.; Allard, S.; Scherf, U.; Cacialli, F. Chem. Commun. 2011, 47, 8820.

(64) Zampetti, A.; Minotto, A.; Squeo, B. M.; Gregoriou, V. G.; Allard, S.; Scherf, U.; Chochos, C. L.; Cacialli, F. Sci. Rep. 2017, 7, 1611.

(65) Sassi, M.; Buccheri, N.; Rooney, M.; Botta, C.; Bruni, F.; Giovanella, U.; Brovelli, S.; Beverina, L. Sci. Rep. 2016, 6, 34096.

(66) Lombeck, F.; Di, D.; Yang, L.; Meraldi, L.; Athanasopoulos, S.; Credgington, D.; Sommer, M.; Friend, R. H. Macromolecules 2016, 49, 9382.

(67) Cacialli, F.; Stoneham, M. J. Phys.: Condens. Matter 2002, 14, V9.
(68) Qian, G.; Zhong, Z.; Luo, M.; Yu, D.; Zhang, Z.; Ma, D.; Wang, Z. Y. J. Phys. Chem. C 2009, 113, 1589.

(69) Ledwon, P.; Zassowski, P.; Jarosz, T.; Lapkowski, M.; Wagner, P.; Cherpak, V.; Stakhira, P. J. Mater. Chem. C 2016, 4, 2219.

(70) Yao, L.; Zhang, S.; Wang, R.; Li, W.; Shen, F.; Yang, B.; Ma, Y. Angew. Chem. Int. Ed. 2014, 53, 2119.

(71) Han, X.; Bai, Q.; Yao, L.; Liu, H.; Gao, Y.; Li, J.; Liu, L.; Liu, Y.; Li, X.; Lu, P.; Yang, B. Adv. Funct. Mater. 2015, 25, 7521.

(72) Ellinger, S.; Graham, K. R.; Shi, P.; Farley, R. T.; Steckler, T. T.; Brookins, R. N.; Taranekar, P.; Mei, J.; Padilha, L. A.; Ensley, T. R. Chem. Mater. 2011, 23, 3805.

(73) Mayerhoffer, U.; Fimmel, B.; Würthner, F. Angew. Chem. Int. Ed. 2012, 51, 164.

(74) Pope, M.; Kallmann, H. P.; Magnante, P. J. Chem. Phys. 1963, 38, 2042.

(75) Delorme, R.; Perrin, F. J. Phys. Radium 1929, 10, 177.

(76) Parker, C. A.; Hatchard, C. G. Trans. Faraday Soc. 1961, 57, 189423.

(77) Parker, C. A.; Joyce, T. A. Chem. Commun. 1968, 1421.

(78) Uoyama, H.; Goushi, K.; Shizu, K.; Nomura, H.; Adachi, C. Nature 2012, 492, 234.

(79) Adachi, C.; Baldo, M. A.; Thompson, M. E.; Forrest, S. R. J. Appl. Phys. 2001, 90, 5048.

(80) Dias, F. B.; Bourdakos, K. N.; Jankus, V.; Moss, K. C.; Kamtekar, K. T.; Bhalla, V.; Santos, J.; Bryce, M. R.; Monkman, A. P. Adv. Mater. 2013, 25, 3707.

(81) Freeman, D. M. E.; Musser, A. J.; Frost, J. M.; Stern, H. L.; Forster, A. K.; Fallon, K. J.; Rapidis, A. G.; Cacialli, F.; McCulloch, I.; Clarke, T. M.; Friend, R. H.; Bronstein, H. J. Am. Chem. Soc. 2017, 139, 11073.

(82) Xue, J.; Li, C.; Xin, L.; Duan, L.; Qiao, J. Chem. Sci. 2016, 7, 2888.

(83) Peng, Q.; Obolda, A.; Zhang, M.; Li, F. Angew. Chem. Int. Ed. 2015, $54,7091$.

(84) Ai, X.; Evans, E. W.; Dong, S.; Gillett, A.J.; Guo, H.; Chen, Y.; Hele, T. J. H.; Friend, R. H.; Li, F. Nature 2018, 563, 536.

(85) Lee, S. Y.; Yasuda, T.; Komiyama, H.; Lee, J.; Adachi, C. Adv. Mater. 2016, 28, 4019.

(86) Sun, K.; Chu, D.; Cui, Y.; Tian, W.; Sun, Y.; Jiang, W. Org. Electron. 2017, 48, 389.

(87) Li, C.; Duan, R.; Liang, B.; Han, G.; Wang, S.; Ye, K.; Liu, Y.; Yi, Y.; Wang, Y. Angew. Chem. Int. Ed. 2017, 56, 11525.

(88) Hu, Y.; Yuan, Y.; Shi, Y.-L.; Lin, J.-D.; Jiang, Z.-Q.; Liao, L.-S. J. Mater. Chem. C 2018, 6, 1407.

(89) Li, J.; Nakagawa, T.; MacDonald, J.; Zhang, Q.; Nomura, H.; Miyazaki, H.; Adachi, C. Adv. Mater. 2013, 25, 3319.

(90) Zeng, W.; Lai, H. Y.; Lee, W. K.; Jiao, M.; Shiu, Y. J.; Zhong, C.; Gong, S.; Zhou, T.; Xie, G.; Sarma, M.; Wong, K. T.; Wu, C. C.; Yang, C. Adv. Mater. 2018, 30, 1704961.

(91) Wang, S.; Yan, X.; Cheng, Z.; Zhang, H.; Liu, Y.; Wang, Y. Angew. Chem. Int. Ed. 2015, 54, 13068.

(92) Yuan, Y.; Hu, Y.; Zhang, Y.-X.; Lin, J.-D.; Wang, Y.-K.; Jiang, Z.-Q.; Liao, L.-S.; Lee, S.-T. Adv. Funct. Mater. 2017, 27, 1700986.

(93) Hu, Y.; Yuan, Y.; Shi, Y.-L.; Li, D.; Jiang, Z.-Q.; Liao, L.-S. Adv. Funct. Mater. 2018, 28, 1802597.

(94) D’Aléo, A.; Sazzad, M. H.; Kim, D. H.; Choi, E. Y.; Wu, J. W.; Canard, G.; Fages, F.; Ribierre, J. C.; Adachi, C. Chem. Commun. 2017, 53, 7003.

(95) Kim, D.-H.; D’Aléo, A.; Chen, X.-K.; Sandanayaka, A. D. S.; Yao, D.; Zhao, L.; Komino, T.; Zaborova, E.; Canard, G.; Tsuchiya, Y.; Choi, E.; Wu, J. W.; Fages, F.; Brédas, J.-L.; Ribierre, J.-C.; Adachi, C. Nat. Photonics 2018, 12, 98. 
(96) Nakanotani, H.; Higuchi, T.; Furukawa, T.; Masui, K.; Morimoto, K.; Numata, M.; Tanaka, H.; Sagara, Y.; Yasuda, T.; Adachi, C. Nat. Commun. 2014, 5, 4016.

(97) Nagata, R.; Nakanotani, H.; Adachi, C. Adv. Mater. 2017, 29, 1604265.

(98) Zhang, D.; Duan, L.; Li, C.; Li, Y.; Li, H.; Zhang, D.; Qiu, Y. Adv. Mater. 2014, 26, 5050.

(99) Xue, J.; Liang, Q.; Zhang, Y.; Zhang, R.; Duan, L.; Qiao, J. Adv. Funct. Mater. 2017, 27, 1703283.

(100) Song, X.; Zhang, D.; Lu, Y.; Yin, C.; Duan, L. Adv. Mater. 2019, 31 , 1901923.

(101) Xiang, H.; Cheng, J.; Ma, X.; Zhou, X.; Chruma, J. J. Chem. Soc. Rev. 2013, 42, 6128.

(102) Kido, J.; Ikeda, W.; Kimura, M.; Nagai, K. Jpn. J. Appl. Phys. 1996, 35, L394.

(103) Wei, H.; Yu, G.; Zhao, Z.; Liu, Z.; Bian, Z.; Huang, C. Dalton Trans. 2013, 42, 8951.

(104) Sun, R. G.; Wang, Y. Z.; Zheng, Q. B.; Zhang, H. J.; Epstein, A. J. J. Appl. Phys. 2000, 87, 7589.

(105) Harrison, B. S.; Foley, T. J.; Bouguettaya, M.; Boncella, J. M.; Reynolds, J. R.; Schanze, K. S.; Shim, J.; Holloway, P. H.; Padmanaban, G.; Ramakrishnan, S. Appl. Phys. Lett. 2001, 79, 3770.

(106) Curry, R. J.; Gillin, W. P.; Knights, A. P.; Gwilliam, R. Appl. Phys. Lett. 2000, 77, 2271

(107) Khreis, O. M.; Curry, R. J.; Somerton, M.; Gillin, W. P. J. Appl. Phys. 2000, 88, 777.

(108) Curry, R. J.; Gillin, W. P. Appl. Phys. Lett. 1999, 75, 1380.

(109) Gillin, W. P.; Curry, R. J. Appl. Phys. Lett. 1999, 74, 798.

(110) Kawamura, Y.; Wada, Y.; Yanagida, S.Jpn.J. Appl. Phys. 2001,40, 350.

(111) Cao, X.; Miao, J.; Zhu, M.; Zhong, C.; Yang, C.; Wu, H.; Qin, J.; Cao, Y. Chem. Mater. 2015, 27, 96.

(112) Baldo, M. A.; O’Brien, D. F.; You, Y.; Shoustikov, A.; Sibley, S.; Thompson, M. E.; Forrest, S. R. Nature 1998, 395, 151.

(113) Baldo, M. A.; Lamansky, S.; Burrows, P. E.; Thompson, M. E.; Forrest, S. R. Appl. Phys. Lett. 1999, 75, 4.

(114) Cleave, V.; Yahioglu, G.; Barny, P. L.; Friend, R. H.; Tessler, N. Adv. Mater. 1999, 11, 285.

(115) Adachi, C.; Baldo, M. A.; Forrest, S. R.; Thompson, M. E. Appl. Phys. Lett. 2000, 77, 904.

(116) Lamansky, S.; Djurovich, P.; Murphy, D.; Abdel-Razzaq, F.; Lee, H. E.; Adachi, C.; Burrows, P. E.; Forrest, S. R.; Thompson, M. E. J. Am. Chem. Soc. 2001, 123, 4304.

(117) Harrison, B. S.; Foley, T. J.; Knefely, A. S.; Mwaura, J. K.; Cunningham, G. B.; Kang, T.-S.; Bouguettaya, M.; Boncella, J. M.; Reynolds, J. R.; Schanze, K. S. Chem. Mater. 2004, 16, 2938.

(118) Tuong Ly, K.; Chen-Cheng, R.-W.; Lin, H.-W.; Shiau, Y.-J.; Liu, S.-H.; Chou, P.-T.; Tsao, C.-S.; Huang, Y.-C.; Chi, Y. Nat. Photonics 2017, 11, 63.

(119) Qiao, J.; Duan, L.; Tang, L.; He, L.; Wang, L.; Qiu, Y.J. Mater. Chem. 2009, 19, 6573.

(120) Tao, R.; Qiao, J.; Zhang, G.; Duan, L.; Chen, C.; Wang, L.; Qiu, Y.J. Mater. Chem. C 2013, 1, 6446.

(121) Kesarkar, S.; Mroz, W.; Penconi, M.; Pasini, M.; Destri, S.; Cazzaniga, M.; Ceresoli, D.; Mussini, P. R.; Baldoli, C.; Giovanella, U.; Bossi, A. Angew. Chem. Int. Ed. 2016, 55, 2714.

(122) Rayabarapu, D. K.; Paulose, B. M. J. S.; Duan, J. P.; Cheng, C. H. Adv. Mater. 2005, 17, 349.

(123) Tao, R.; Qiao, J.; Zhang, G.; Duan, L.; Wang, L.; Qiu, Y. J. Phys. Chem. C 2012, 116, 11658.

(124) Xin, L.; Xue, J.; Lei, G.; Qiao, J. RSC Advances 2015, 5, 42354.
(125) Nisic, F.; Colombo, A.; Dragonetti, C.; Roberto, D.; Valore, A.; Malicka, J. M.; Cocchi, M.; Freeman, G. R.; Williams, J. A. G. J. Mater. Chem. C 2014, 2, 1791.

(126) Freeman, D. M. E.; Minotto, A.; Duffy, W.; Fallon, K. J.; McCulloch, I.; Cacialli, F.; Bronstein, H. Polym. Chem. 2016, 7, 722.

(127) Cocchi, M.; Virgili, D.; Fattori, V.; Williams, J. A. G.; Kalinowski, J. Appl. Phys. Lett. 2007, 90, 023506.

(128) Cocchi, M.; Kalinowski, J.; Virgili, D.; Williams, J. A. G. Appl. Phys. Lett. 2008, 92, 113302.

(129) Williams, E. L.; Li, J.; Jabbour, G. E. Appl. Phys. Lett. 2006, 89, 083506.

(130) Ho, C.-L.; Yao, B.; Zhang, B.; Wong, K.-L.; Wong, W.-Y.; Xie, Z.; Wang, L.; Lin, Z. Organomet. Chem. 2013, 730, 144.

(131) Tsuzuki, T.; Tokito, S. Adv. Mater. 2007, 19, 276.

(132) Xue, J.; Xin, L.; Hou, J.; Duan, L.; Wang, R.; Wei, Y.; Qiao, J. Chem. Mater. 2017, 29, 4775.

(133) Lee, T.-C.; Hung, J.-Y.; Chi, Y.; Cheng, Y.-M.; Lee, G.-H.; Chou, P.-T.; Chen, C.-C.; Chang, C.-H.; Wu, C.-C. Adv. Funct. Mater. 2009, 19, 2639.

(134) Liao, J. L.; Chi, Y.; Liu, S. H.; Lee, G. H.; Chou, P. T.; Huang, H. X.; Su, Y. D.; Chang, C. H.; Lin, J. S.; Tseng, M. R. Inorg. Chem. 2014, 53, 9366.

(135) Tessler, N.; Medvedev, V.; Kazes, M.; Kan, S.; Banin, U. Science 2002, 295, 1506.

(136) Bansal, A. K.; Antolini, F.; Zhang, S.; Stroea, L.; Ortolani, L.; Lanzi, M.; Serra, E.; Allard, S.; Scherf, U.; Samuel, I. D. W.J. Phys. Chem. C 2016, 120, 1871.

(137) Cheng, K. Y.; Anthony, R.; Kortshagen, U. R.; Holmes, R. J. Nano Lett. 2011, 11, 1952.

(138) Sun, L.; Choi,J.J.; Stachnik, D.; Bartnik, A.C.; Hyun, B. R.; Malliaras, G. G.; Hanrath, T.; Wise, F. W. Nat. Nanotechnol. 2012, 7, 369.

(139) Yan, L.; Shen, X.; Zhang, Y.; Zhang, T.; Zhang, X.; Feng, Y.; Yin, J.; Zhao, J.; Yu, W. RSC Advances 2015, 5, 54109.

(140) Ma, X.; Xu, F.; Benavides, J.; Cloutier, S. G. Org. Electron. 2012, 13, 525.

(141) Yang, Z.; Voznyy, O.; Liu, M.; Yuan, M.; Ip, A. H.; Ahmed, O. S.; Levina, L.; Kinge, S.; Hoogland, S.; Sargent, E. H. ACS Nano 2015 , 9, 12327.

(142) Supran, G. J.; Song, K. W.; Hwang, G. W.; Correa, R. E.; Scherer, J.; Dauler, E. A.; Shirasaki, Y.; Bawendi, M. G.; Bulović, V. Adv. Mater. 2015, 27, 1437.

(143) Bourdakos, K. N.; Dissanayake, D. M. N. M.; Lutz, T.; Silva, S. R. P.; Curry, R. J. Appl. Phys. Lett. 2008, 92, 153311.

(144) Choudhury, K. R.; Song, D. W.; So, F. Org. Electron. 2010, 11, 23.

(145) Gong, X.; Yang, Z.; Walters, G.; Comin, R.; Ning, Z.; Beauregard, E.; Adinolfi, V.; Voznyy, O.; Sargent, E. H. Nat. Photonics 2016, 10, 253.

(146) Hu, W.; Henderson, R.; Zhang, Y.; You, G.; Wei, L.; Bai, Y.; Wang, J.; Xu, J. Nanotechnology 2012, 23, 375202.

(147) Qiu, W.; Xiao, Z.; Roh, K.; Noel, N. K.; Shapiro, A.; Heremans, P.; Rand, B. P. Adv. Mater. 2019, 31, 1806105.

(148) Chiba, T.; Hayashi, Y.; Ebe, H.; Hoshi, K.; Sato, J.; Sato, S.; Pu, Y.J.; Ohisa, S.; Kido, J. Nat. Photonics 2018, 12, 681.

(149) Lin, K.; Xing, J.; Quan, L. N.; de Arquer, F. P. G.; Gong, X.; Lu, J.; Xie, L.; Zhao, W.; Zhang, D.; Yan, C.; Li, W.; Liu, X.; Lu, Y.; Kirman, J.; Sargent, E. H.; Xiong, Q.; Wei, Z. Nature 2018, 562, 245.

(150) Cao, Y.; Wang, N.; Tian, H.; Guo, J.; Wei, Y.; Chen, H.; Miao, Y.; Zou, W.; Pan, K.; He, Y.; Cao, H.; Ke, Y.; Xu, M.; Wang, Y.; Yang, M.; Du, K.; Fu, Z.; Kong, D.; Dai, D.; Jin, Y.; Li, G.; Li, H.; Peng, Q.; Wang, J.; Huang, W. Nature 2018, 562, 249. 
(151) Wang, N.; Cheng, L.; Ge, R.; Zhang, S.; Miao, Y.; Zou, W.; Yi, C.; Sun, Y.; Cao, Y.; Yang, R.; Wei, Y.; Guo, Q.; Ke, Y.; Yu, M.; Jin, Y.; Liu, Y.; Ding, Q.; Di, D.; Yang, L.; Xing, G.; Tian, H.; Jin, C.; Gao, F.; Friend, R. H.; Wang, J.; Huang, W. Nat. Photonics 2016, 10, 699.

(152) Gil-Escrig, L.; Longo, G.; Pertegás, A.; Roldán-Carmona, C.; Soriano, A.; Sessolo, M.; Bolink, H. J. Chem. Commun. 2015, 51, 569.

(153) Kumawat, N. K.; Dey, A.; Narasimhan, K. L.; Kabra, D. ACS Photonics 2015, 2, 349.

(154) Wang, J.; Wang, N.; Jin, Y.; Si, J.; Tan, Z. K.; Du, H.; Cheng, L.; Dai, X.; Bai, S.; He, H.; Ye, Z.; Lai, M. L.; Friend, R. H.; Huang, W. Adv. Mater. 2015, 27, 2311.

(155) Hong, W. L.; Huang, Y. C.; Chang, C. Y.; Zhang, Z. C.; Tsai, H. R.; Chang, N. Y.; Chao, Y. C. Adv. Mater. 2016, 28, 8029.

(156) Lai, M. L.; Tay, T. Y.; Sadhanala, A.; Dutton, S. E.; Li, G.; Friend, R. H.; Tan, Z. K. J. Phys. Chem. Lett. 2016, 7, 2653.
(157) Matterson, B. J.; Lupton, J. M.; Safonov, A. F.; Salt, M. G.; Barnes, W. L.; Samuel, I. D. W. Adv. Mater. 2001, 13, 123.

(158) Ziebarth, J. M.; Saafir, A. K.; Fan, S.; McGehee, M. D. Adv. Funct. Mater. 2004, 14, 451.

(159) Sun, Y.; Forrest, S. R. Nat. Photonics 2008, 2, 483.

(160) Giuri, A.; Yuan, Z.; Miao, Y.; Wang, J.; Gao, F.; Sestu, N.; Saba, M.; Bongiovanni, G.; Colella, S.; Esposito Corcione, C.; Gigli, G.; Listorti, A.; Rizzo, A. Sci. Rep. 2018, 8, 15496.

(161) Xiao, Z.; Kerner, R. A.; Zhao, L.; Tran, N. L.; Lee, K. M.; Koh, T.-W.; Scholes, G. D.; Rand, B. P. Nat. Photonics 2017, 11, 108.

(162) Zhao, L.; Yeh, Y. W.; Tran, N. L.; Wu, F.; Xiao, Z.; Kerner, R. A.; Lin, Y. L.; Scholes, G. D.; Yao, N.; Rand, B. P. ACS Nano 2017, 11, 3957.

(163) Yuan, M.; Quan, L. N.; Comin, R.; Walters, G.; Sabatini, R.; Voznyy, O.; Hoogland, S.; Zhao, Y.; Beauregard, E. M.; Kanjanaboos, P.; Lu, Z.; Kim, D. H.; Sargent, E. H. Nat. Nanotechnol. 2016, 11, 872. 